\title{
POLYHEDRAL CONVEX CONES AND THE EQUATIONAL THEORY OF THE BICYCLIC SEMIGROUP
}

\author{
F. PASTIJN
}

(Received 2 December 2004; revised 8 April 2005)

Communicated by D. Easdown

\begin{abstract}
To any given balanced semigroup identity $v \approx w$ a number of polyhedral convex cones are associated. In this setting an algorithm is proposed which determines whether the given identity is satisfied in the bicylic semigroup

$$
B C=\left\langle a, b \mid a^{2} b=a b a=a, a b^{2}=b a b=b\right\rangle
$$

or in the semigroup

$$
E=\left\langle a, b \mid a^{2} b=a, a b^{2}=b\right\rangle .
$$

The semigroups $B C$ and $E$ deserve our attention because a semigroup variety contains a simple semigroup which is not completely simple (respectively, which is idempotent free) if and only if this variety contains $B C$ (respectively, $E$ ). Therefore, for a given identity $v \approx w$ it is decidable whether or not the variety determined by $v \approx w$ contains a simple semigroup which is not completely simple (respectively, which is idempotent free).
\end{abstract}

2000 Mathematics subject classification: primary 20M05, $20 \mathrm{M} 07$.

\section{Introduction}

One of the main issues of [19] was to characterize and classify the semigroup varieties satisfying the property $[\mathcal{J}=\mathcal{D}$ ], that is, the semigroup varieties consisting of semigroups where the Green relations $\mathcal{J}$ and $\mathcal{D}$ coincide. All the varieties minimal for not satisfying $[\mathcal{J}=\mathcal{D}]$ were found. After this, it is only natural to ask for a characterization of the semigroup varieties in which all the simple semigroups are bisimple. That this is a much weaker condition is testified by the fact that even in the join of all the semigroup varieties minimal for not satisfying $[\mathcal{J}=\mathcal{D}]$, all the simple semigroups are completely simple (concluding remark of Section 4).

(C) 2006 Australian Mathematical Society $1446-7887 / 06 \$ A 2.00+0.00$ 
From the investigations by Jones [12] it follows that a semigroup variety contains a simple semigroup which is not bisimple if and only if it contains the bicyclic semigroup $B C$ (Corollary 2.5 ). It is for this reason that we carefully examine the equational theory of $B C$. We determine whether a given identity belongs to the equational theory of $B C$ in three distinct ways (Theorems $4.1,4.5,4.6$ ). The last characterization is one of determinantal form which uses a consistency criterion, due to Motzkin [17], for a system of strict inequalities. Polyhedral convex cones that can be derived from the given identity in a way explained in Section 3 provide an adequate setting for formulating these results.

A sufficient condition (Theorem 4.10) enables us to generate, with relative ease, examples of identities which are satisfied in $B C$ (Theorem 4.14). As a result, we are able to improve on the celebrated result by Shneerson [24] which states that the variety generated by $B C$ is of infinite axiomatic rank (Theorem 5.5, Corollary 5.6).

From [12] it also follows that a semigroup variety contains an idempotent free simple semigroup if and only if it contains the idempotent free simple semigroup $E$ (Corollary 2.3). The variety generated by $E$ contains the variety generated by $B C$ properly, in fact, the interval in the lattice of semigroup varieties between the varieties generated by $B C$ and by $E$ respectively, is of the power of the continuum (Theorem 6.3). Again, using the language of polyhedral convex cones, we characterize the identities which belong to the equational theory of $E$ (Theorem 6.4). A sufficient condition (Theorem 6.8) that requires some of the polyhedral convex cones involved to coincide with their boundary, enables us to generate plenty of identities satisfied in $E$ (Theorem 6.9).

\section{Preliminaries}

We refer to $[8,11]$ for the necessary background on semigroups, and in particular on (0-)simple, (0-)bisimple, and completely (0-)simple semigroups. For a general background on universal algebra we refer to [14]. It remains to review some further terminology and notation which will be used in this paper.

In the following $X$ will be a fixed countably infinite set, the elements of which we call variables. These variables will be denoted by $x, y, x^{\prime}, y_{i}, \ldots$, and the like. For any nonempty set $A, A^{*}\left(A^{+}\right)$will be the set of all (nonempty) words whose letters belong to $A$. Then $A^{+}\left(A^{*}\right)$ forms a semigroup (with identity) for the operation of catenation of words. For any $v \in X^{+}$and $x \in X, n_{x}(v)$ denotes the number of occurrences of $x$ in $v$. Then $c(v)=\left\{x \in X \mid n_{x}(v) \geq 1\right\}$ is called content of $v$ and $\ell(v)=\sum_{x \in c(v)} n_{x}(v)$ the length of $v$. If 1 denotes the empty word of $X^{*}$ we agree to put $c(1)=\emptyset$ and $\ell(1)=0$. For $v \in X^{+}$and $v=v_{1} v_{2}$ in $X^{*}$, we call $v_{1}$ a prefix of $v$ and $v_{2}$ a suffix of $v$. For $v \in X^{+}, h(v)(t(v))$ denotes the first (last) letter of $v$. 
Let $S$ be a semigroup. A mapping $\sigma: X \rightarrow S$ will be called a substitution by elements of $S$. This mapping extends in a unique way to a homomorphism of $X^{+}$ into $S$, and we agree to denote this homomorphism again by $\sigma$. A semigroup identity is a pair of words of $X^{+}$, commonly denoted in the form $v \approx w$, where $v, w \in X^{+}$. We say that the identity $v \approx w$ is satisfied in $S$ if $v \sigma=w \sigma$ holds true in $S$ for every substitution $\sigma$ by elements of $S$. The identity $v \approx w$ is called balanced if $n_{x}(v)=n_{x}(w)$ for all $x \in X$. If this is the case, then $\ell(v)=\ell(w)$, and $\ell(v)=\ell(w)$ will be called the length of $v \approx w$. An identity is satisfied in the infinite cyclic semigroup if and only if it is balanced.

Let $\Sigma$ be a nonempty set of identities. We say that the identity $v \approx w$ is a consequence of $\Sigma$ if there exists a positive integer $k$, words $u_{1}, \ldots, u_{k} \in X^{+}$, and substitutions $\sigma_{1}, \ldots, \sigma_{k-1}$ by elements of $X^{+}$such that $v=u_{1}, u_{k}=w$, and for all $1 \leq i<k$,

$$
\begin{aligned}
u_{i} & =r_{i}\left(p_{i} \sigma_{i}\right) s_{i}, \\
u_{i+1} & =r_{i}\left(q_{i} \sigma_{i}\right) s_{i},
\end{aligned}
$$

for some $r_{i}, s_{i} \in X^{*}$ and $p_{i}, q_{i} \in X^{+}$, where $p_{i} \approx q_{i}$ or $q_{i} \approx p_{i}$ belongs to $\Sigma$. An equational theory is a set of identities which is closed for taking consequences. If $S$ is a semigroup, then the set of all identities that are satisfied in $S$ is an equational theory and will be called the equational theory of $S$.

Let $\Sigma$ be a equational theory. A word $v \in X^{+}$is called an isoterm of $\Sigma$ if, whenever $v \approx w$ belongs to $\Sigma, v=w$. If $A$ is a subset of $\Sigma$ such that $\Sigma$ consists of all the consequences of $A$, then $A$ is called a basis of $\Sigma$. $\Sigma$ is said to be of finite axiomatic rank if there exists a positive integer $n$ and a basis $A$ of $\Sigma$ where the number of variables involved in each identity of $A$ is less than $n$; otherwise $\Sigma$ is said to be of infinite axiomatic rank. With some abuse of terminology we shall permit ourselves to say that, for a given basis $A$ of $\Sigma$, the identity $p \approx q$ of $\Sigma$ belongs to $A$ if for some permutation $\sigma$ of $X, p \sigma \approx q \sigma$ or $q \sigma \approx p \sigma$ is an element of $A$.

For any class $K$ of semigroups, let $\mathbf{H}(K)(\mathbf{S}(K))$ be the class of all semigroups which are isomorphic to a homomorphic image (a subsemigroup) of a member of $\mathbf{K}$, and $\mathbf{P}(\mathbf{K})$ the class of all semigroups which are isomorphic to the direct product of members of $\mathbf{K}$. The class $\mathbf{K}$ is said to be a variety if $\mathbf{K}$ is closed for the class operators $\mathbf{H}, \mathbf{S}$, and $\mathbf{P}$, that is, $\mathbf{K}=\mathbf{H}(\mathbf{K})=\mathbf{S}(\mathbf{K})=\mathbf{P}(\mathbf{K})$. For any class $\mathbf{K}, \mathbf{H S P}(K)$ is the smallest variety containing $K$. If $K$ consists of the semigroup $S$ only, then $\operatorname{HSP}(K)$ will also be denoted $\operatorname{HSP}(S)$. An equational class of semigroups is a class consisting of all the semigroups in which all the identities of an equational theory are satisfied. By Birkhoff's completeness theorem, the notions of variety and equational class coincide. In particular, for any semigroup $S, \mathbf{H S P}(S)$ consists of all the semigroups in which all the identities satisfied by $S$ are satisfied. Accordingly, $\operatorname{HSP}(S)$ will be said to be of finite (infinite) axiomatic rank if the same is true for the equational theory of $S$. 


\section{The significance of the semigroups $A, E$, and $B C$}

The semigroups $A, E$, and $B C$ are each generated by two generators, say $a$ and $b$. They have the following presentations:

$$
\begin{aligned}
A & =\left\langle a, b \mid a^{2} b=a\right\rangle, \\
E & =\left\langle a, b \mid a^{2} b=a, a b^{2}=b\right\rangle, \\
B C & =\left\langle a, b \mid a^{2} b=a b a=a, a b^{2}=b a b=b\right\rangle .
\end{aligned}
$$

Clearly then, $E$ is a homomorphic image of $A$, and $B C$ is a homomorphic image of $E$, hence $\operatorname{HSP}(B C) \subseteq \operatorname{HSP}(E) \subseteq \operatorname{HSP}(A)$. We shall soon discover that these inclusions are strict.

It is fairly easy to solve the word problem for each of these three presentations. It was noticed in [12, Theorem 2.7 (i)] that every element of $A$ can be written in a unique way in the form $v(a b)^{\ell} a^{m}$, with $v$ a word over $\left\{(a b)^{n-1} b \mid n \geq 2\right\}, \ell \geq 0, m \geq 0$, and with $v$ nonempty if $\ell=m=0$. Moreover, the subsemigroup of $A$ generated by the elements of the form $(a b)^{n-1} b, n \geq 1$, is actually freely generated by those elements $[12$, Theorem 2.7 (vi)], and therefore $\operatorname{HSP}(A)=\mathbf{S}$, the variety of all semigroups.

RESULT 2.1 (see [12]). A right simple semigroup which is not a right group generates the variety $\mathbf{S}$ of all semigroups.

PrOOF. Let $S$ be a right simple semigroup, which is not a right group. Then $S$ is necessarily idempotent free and by [12, Corollary 4.3] $S$ contains a copy of $A$ as a subsemigroup. Therefore $S$ contains a subsemigroup isomorphic to the free semigroup of infinite rank, whence $\operatorname{HSP}(S)=\mathbf{S}$.

The Croisot-Teissier semigroups and the Baer-Levi semigroups, which were investigated in [8, Chapter 8], form examples of right simple semigroups which are not right groups. The semigroup $A$ has a unique least ideal, namely the ideal consisting of all the elements of $A$ which are not of the form $b^{n}$ for some $n>1$. This ideal is a simple idempotent free semigroup [12, Theorem $2.7(\mathrm{v})]$, but since it contains a free semigroup of infinite rank, it generates the variety $\mathbf{S}$ of all semigroups. Other interesting examples of simple idempotent free semigroups were given in [3] (see also [8, Exercises 2.1 .10 and 8.4 .6$]$ ), while $[4,5,6]$ provide for further clever constructions of such semigroups. In fact, every idempotent free semigroup can be embedded into a simple idempotent free semigroup [25]. More often than not however, a simple semigroup obtained from these examples and constructions obviously generates the variety of all semigroups. Therefore the following question imposes itself quite naturally: is there any simple idempotent free semigroup which does not generate the variety of all 
semigroups? To answer this question we turn to the semigroup $E$. This semigroup is a special instance of the construction given in [5] (see [12, Example 2.5]).

One readily verifies that every element of the semigroup $E$ can be written uniquely in the form $b^{s}(a b)^{m} a^{n}$ for some $s \geq 0, m \geq 0, n \geq 0$, with $s+m+n \geq 1$. The multiplication in $E$ then follows the following rule: for $b^{s_{1}}(a b)^{m_{1}} a^{n_{1}} \in E$ and $b^{\varsigma_{2}}(a b)^{m_{2}} a^{n_{2}} \in E$, we have

$$
b^{s_{1}}(a b)^{m_{1}} a^{n_{1}} b^{s_{2}}(a b)^{m_{2}} a^{n_{2}}= \begin{cases}b^{s_{1}+s_{2}-n_{1}}(a b)^{m_{2}} a^{n_{2}} & \text { if } s_{2}>n_{1}, \\ b^{s_{1}}(a b)^{m_{1}} a^{n_{1}+n_{2}-s_{2}} & \text { if } n_{1}>s_{2} \\ b^{s_{1}}(a b)^{m_{1}+m_{2}+1} a^{n_{2}} & \text { if } n_{1}=s_{2}>0 \\ b^{s_{1}}(a b)^{m_{1}+m_{2}} a^{n_{2}} & \text { if } n_{1}=s_{2}=0\end{cases}
$$

Clearly, $E$ is idempotent free. For any $b^{s}(a b)^{m} a^{n} \in E$ we have that

$$
a^{s+2}\left(b^{s}(a b)^{m} a^{n}\right) b^{n+1}=a,
$$

whence $E$ is simple. For the sake of completeness we should mention that the semigroup $E$ was investigated for various reasons in [12, 15, 21, 22].

Recall that by an idempotent free 0 -simple semigroup we mean a 0 -simple semigroup that does not contain an idempotent other than 0 .

RESULT 2.2 (see [12]). A (0-)simple idempotent free semigroup which does not generate the variety of all semigroups contains a copy of $E$ as a subsemigroup.

ProOF. From [12, Theorems 4.2, 6.4 and the concluding remark] it follows that a 0 -simple idempotent free semigroup contains a copy of the free semigroup of infinite rank or a copy of $E$ as a subsemigroup.

COROLLARY 2.3. HSP $(E)$ is the smallest variety containing a (0-)simple idempotent free semigroup.

Of course the statements of Result 2.2 and Corollary 2.3 would be self-evident if $E$ does not generate a proper semigroup variety. Later in this paper we shall indeed find an infinity of nontrivial semigroup identities, which are satisfied in $E$. In the meantime we take heart from the fact that no free semigroup of rank greater than 1 divides $E$ [12, Theorem 2.3 and subsequent remark].

The semigroup $B C$ is called the bicyclic semigroup. From the presentation of $B C$ it follows that the element $a b$ of $B C$ is the identity element of $B C$. If we agree to write $a b=1=a^{0}=b^{0}$, then we have that every element of $B C$ can be written uniquely in the form $b^{5} a^{n}$ for some $s \geq 0, n \geq 0$. Further, for any $b^{s_{1}} a^{n_{1}} \in B C$ and 
$b^{s_{2}} a^{n_{2}} \in B C$ we have

$$
b^{s_{1}} a^{n_{1}} b^{s_{2}} a^{n_{2}}= \begin{cases}b^{s_{1}+s_{2}-n_{1}} a^{n_{2}} & \text { if } s_{2} \geq n_{1}, \\ b^{s_{1}} a^{n_{1}+n_{2}-s_{2}} & \text { if } n_{1} \geq s_{2} .\end{cases}
$$

From (2.1) and (2.2) it now follows that

$$
E \rightarrow B C, \quad b^{s}(a b)^{m} a^{n} \rightarrow b^{s} a^{n}
$$

is a homomorphism from $E$ onto $B C$. Since $E$ is simple, so is its homomorphic image $B C$. The semigroup $B C$ is not idempotent free; the idempotents of $B C$ are precisely the elements of the form $b^{s} a^{s}, s \geq 0$.

RESULT 2.4 (see [3]). A (0-)simple semigroup which is not idempotent free and is not completely (0-)simple contains a copy of $B C$ as a subsemigroup.

The proof of the above result can also be found in [8, Theorem 2.54].

COROLLARY 2.5. For a semigroup variety $\mathbf{V}$, the following are equivalent:

(i) V contains $B C$,

(ii) $\mathbf{V}$ contains a simple semigroup which is not bisimple,

(iii) V contains a (0-)simple semigroup which is not completely (0-)simple.

PROOF. The subsemigroup of $B C$ which consists of the elements $b^{s} a^{n}, s \geq 0$, $n \geq 0$, with either $s$ and $n$ both even or $s$ and $n$ both odd, is simple but not bisimple since it consists of two $\mathcal{D}$-classes (see also [18]). Therefore (i) implies (ii). Since every completely (0-)simple semigroup is (0-)bisimple, we have that (ii) implies (iii). Let us now assume that $\mathbf{V}$ satisfies (iii). If $\mathbf{V}$ contains a (0-)simple idempotent free semigroup, then $\mathbf{V}$ contains $E$ by Corollary 2.3 , and therefore $\mathbf{V}$ also contains the homomorphic image $B C$ of $E$. Otherwise, $\mathbf{V}$ contains $B C$ by Result 2.4. Thus, (iii) implies (i).

Corollaries 2.3 and 2.5 provide us with the necessary motivation for investigating the equational theories of $E$ and of $B C$. Since the equational theory of $E$ is contained in the equational theory of $B C$, the latter one will be studied first.

One of the major issues of [19] was the classification of the semigroup varieties which satisfy the property $[\mathcal{J}=\mathcal{D}]$, that is, the semigroup varieties which consist of semigroups for which the Green relations $\mathcal{J}$ and $\mathcal{D}$ coincide. In such varieties simple semigroups must be bisimple, and it is precisely because of our present Corollary 2.5 that the variety generated by $B C$ was eyed with special concern. In [19] the varieties minimal for not satisfying $[\mathcal{J}=\mathcal{D}]$ were found; they are countably infinite in number, and every semigroup variety not satisfying $[\mathcal{J}=\mathcal{D}]$ contains one of these minimal 
ones. In view of Corollary 2.5 we know that the semigroup variety $\operatorname{HSP}(B C)$ contains at least one of the varieties minimal for not satisfying $[\mathcal{J}=\mathcal{D}]$. In Section 4 we shall see that $\mathbf{H S P}(B C)$ properly contains the join of all the varieties minimal for not satisfying $[\mathcal{J}=\mathcal{D}]$. The basic strategy in the present paper and [19] is the same. Results 2.2 and 2.4 show that $E$ and $B C$ serve a role as forbidden divisors in a sense explained in full in [19]. The present paper is otherwise quite independent from [19].

We shall henceforth prefer to write the elements of $E$ in the form $b^{s}(a b)^{m} a^{\ell+s \text {, }}$ where $s \geq 0, m \geq 0, \ell$ an integer such that $\ell+s \geq 0$, and such that $\ell+m+2 s \geq 1$. Accordingly, we write the elements of $B C$ in the form $b^{5} a^{\ell+s}$, where $s \geq 0$ and $\ell+s \geq 0$, with $\ell$ an integer. The reason for introducing such integers $\ell$ will be apparent from the following observation.

RESULT 2.6 (see $[12,24])$. The mappings

$$
\begin{aligned}
E & \rightarrow Z, & b^{s}(a b)^{m} a^{\ell+s} & \rightarrow \ell, \\
B C & \rightarrow Z, & b^{s} a^{\ell+s} & \rightarrow \ell,
\end{aligned}
$$

are homomorphisms onto the additive group of the integers.

It is now necessary to introduce some auxiliary results of a technical nature for later consideration. Their purpose is to generalize to any number of factors the rules (2.1) and (2.2) which describe the multiplication in $E$ and $B C$, respectively.

Let $v$ be a nonempty word over $X$,

$$
v=x_{i_{1}} x_{i_{2}} \cdots x_{i_{n}}, \quad c(v)=\left\{x_{1}, \ldots, x_{t}\right\}, \quad n \geq 1,
$$

and let $\varphi$ be a substitution of the variables which occur in $v$ by elements of $B C$

$$
x_{i} \varphi=b^{s_{i}} a^{\ell_{1}+s_{i}}, \quad 1 \leq i \leq t, \quad s_{i} \geq 0, \quad \ell_{i}+s_{i} \geq 0 .
$$

LEMMA 2.7. Let $v$ be a nonempty word over $X$ as in (2.5) and $\varphi$ the substitution (2.6) of the variables which occur in $v$ by elements of $B C$. Let

$$
s=\max \left(s_{i_{1}},-\ell_{i_{1}}+s_{i_{2}}, \ldots,-\ell_{i_{1}}-\ell_{i_{2}}-\cdots-\ell_{i_{n-1}}+s_{i_{n}}\right) .
$$

Then in $B C, v \varphi=b^{s} a^{\ell_{\ell_{1}}+\cdots+\ell_{i_{n}}+s}$.

ProOf. We let $1 \leq j \leq n$ and $v_{j}$ be the prefix of length $j$ of $v$. We show by induction on $j$ that the statement of the lemma holds true for $v_{j}$.

The case $j=1$ is trivial. We next consider the case $j=2$. From Result 2.6 we know that $\left(b^{s_{i_{1}}} a^{\ell_{i_{1}}+s_{i_{1}}}\right)\left(b^{s_{2}} a^{\ell_{i_{2}}+s_{i_{2}}}\right)$ is of the form $b^{t} a^{\ell_{i_{1}}+\ell_{i_{2}}+t}$ for some $t$. From (2.2) we see that if $s_{i_{2}} \geq \ell_{i_{1}}+s_{i_{1}}$, then

$$
t=s_{i_{1}}+s_{i_{2}}-\left(\ell_{i_{1}}+s_{i_{1}}\right)=s_{i_{2}}-\ell_{i_{1}}=\max \left(s_{i_{1}},-\ell_{i_{1}}+s_{i_{2}}\right),
$$


and if $s_{i_{2}} \leq \ell_{i_{1}}+s_{i_{1}}$, then $t=s_{i_{1}}=\max \left(s_{i_{1}},-\ell_{i_{1}}+s_{i_{2}}\right)$. Therefore the statement of the lemma holds true for $v_{2}$.

We next assume that $2 \leq j<n$ and $v_{j} \varphi=b^{t} a^{\ell_{1}+\cdots+\ell_{i j}+t}$, with

$$
t=\max \left(s_{i_{1}},-\ell_{i_{1}}+s_{i_{2}}, \ldots,-\ell_{i_{1}}-\cdots-\ell_{i_{j-1}}+s_{i_{j}}\right) .
$$

Then, using the fact that the statement of the lemma holds true for words of length 2 , we find

$$
\begin{aligned}
v_{j+1} \varphi & =\left(v_{j} \varphi\right)\left(x_{i_{j+1}} \varphi\right) \\
& =b^{t} a^{\ell_{i_{1}}+\cdots+\ell_{i_{j}}+r} b^{s_{j+1}} a^{\ell_{i_{j+1}}+s_{i_{j+1}}}=b^{r} a^{\ell_{i_{i}}+\cdots+\ell_{i_{j+1}}+r},
\end{aligned}
$$

where

$$
\begin{aligned}
r & =\max \left(t,-\left(\ell_{i_{1}}+\cdots+\ell_{i_{j}}\right)+s_{i_{j+1}}\right) \\
& =\max \left(s_{i_{1}},-\ell_{i_{1}}+s_{i_{2}}, \ldots,-\ell_{i_{1}}-\cdots-\ell_{i_{j}}+s_{i_{j+1}}\right)
\end{aligned}
$$

and we see that the statement of the lemma holds true for $v_{j+1}$. This concludes our proof by induction.

If, given a balanced identity $v \approx w$ one wants to verify whether this identity is satisfied in $B C$, one has to try out all possible substitutions by elements of $B C$. The reader who has been experimenting with this type of exercise must at least have had the intuitive feeling that it is sufficient to verify a finite number of cases, and so it should be decidable whether the given $v \approx w$ belongs to be equational theory of $B C$. Lemma 2.7 already makes this more precise; the cases to be considered are related to the circumstance of which entries in (2.7) yield the maximum, and similarly for $w$ instead of $v$.

We shall now do similar work for the semigroup $E$. Let $v$ be as in (2.5), but we now consider a substitution of the variables which occur in $v$ by elements of $E$ :

$$
\begin{gathered}
x_{i} \varphi=b^{s_{i}}(a b)^{m_{i}} a^{\ell_{i}+s_{i}}, \quad i \leq i \leq t, \\
s_{i} \geq 0, \quad m_{i} \geq 0, \quad \ell_{i}+s_{i} \geq 0, \quad \ell_{i}+m_{i}+2 s_{i} \geq 1 .
\end{gathered}
$$

LEMMA 2.8. Let $v$ be a nonempty word over $X$ as in (2.5) and $\varphi$ the substitution (2.8) of the variables which occur in $v$ by elements of $E$. Let $s$ be given by (2.7),

$$
\begin{aligned}
Y & =\left\{j \mid s=-\ell_{i_{1}}-\cdots-\ell_{i_{j-1}}+s_{i_{j}}\right\}, \\
r & =\min Y, \quad Y^{\prime}=\left\{j \in Y \mid s_{i_{j}} \neq 0, j>r\right\}, \quad \text { and } \\
m & =\left(\sum_{j \in Y} m_{i_{j}}\right)+\left|Y^{\prime}\right| .
\end{aligned}
$$

Then in $E, v \varphi=b^{s}(a b)^{m} a^{\ell_{i_{1}}+\cdots+\ell_{i_{n}}+s}$. 
ProOF. Since (2.3) is a homomorphism from $E$ onto $B C$, it follows from Lemma 2.8 that $v \varphi=b^{s}(a b)^{m} a^{k_{1}+\cdots+\ell_{\text {in }}+s}$ for some $m$, and with $s$ given by (2.7). It remains to verify that $m$ is indeed as in the statement of the lemma. The same remark applies to all the prefixes of $v$.

We prove by induction on $i$ that the statement of the lemma holds true for the prefix $v_{r+i}$ of length $r+i$ of $v$, for $0 \leq i \leq n-r$.

We first consider the case $i=0$. If $r=1$, then $v_{r}=x_{i_{1}}, v_{r} \varphi=b^{s_{i_{1}}}(a b)^{m_{i_{1}}} a^{\ell_{i_{1}}+s_{i_{1}}}$ and we see that the exponent $m_{i}$ is according to the calculation in (2.9). So $v_{r} \varphi$ is obviously of the required form. We next assume that $r>1$ and

$$
t=\max \left(s_{i_{1}}, \ldots,-\ell_{i_{1}}-\cdots-\ell_{i_{r-2}}+s_{i_{r-1}}\right) .
$$

Since $r=\min Y$ we know that $t<s$, where

$$
s=\max \left(s_{i_{1}}, \ldots,-\ell_{i_{1}}-\cdots-\ell_{i_{r-1}}+s_{i_{r}}\right)=-\ell_{i_{1}}-\cdots-\ell_{i_{r-1}}+s_{i_{r}} .
$$

Consequently, $t+\ell_{i_{1}}+\cdots+\ell_{i_{r-1}}<s_{i_{r}}$. From the remark made before we have that

$$
v_{r-1} \varphi=b^{t}(a b)^{m^{\prime}} a^{\ell_{i_{1}}+\cdots+\ell_{i_{r-1}}+t}
$$

for some $m^{\prime}$. Therefore, applying $a b^{2}=b$ repeatedly we obtain

$$
\begin{aligned}
v_{r} \varphi & =\left(v_{r-1} \varphi\right)\left(x_{i_{r}} \varphi\right)=b^{\prime}(a b)^{m^{\prime}} a^{\ell_{i_{1}}+\cdots+\ell_{i_{r-1}}+t} b^{s_{i r}}(a b)^{m_{i_{r}}} a^{\ell_{i_{r}}+s_{i_{r}}} \\
& =b^{t+s_{t_{r}}-\left(\ell_{\ell_{1}}+\cdots+\ell_{i_{r}-1}+t\right)}(a b)^{m_{i_{r}}} a^{\ell_{i_{r}}+s_{i_{r}}}=b^{s}(a b)^{m_{i_{r}}} a^{\ell_{i_{1}}+\cdots+\ell_{i_{r}}+s}
\end{aligned}
$$

and again $v_{r} \varphi$ is of the required form.

We now consider $i$ such that $r \leq r+i<n$ and we assume that the statement of the lemma holds true for $v_{r+i}$. In other words, we assume that

$$
v_{r+i} \varphi=b^{s}(a b)^{m^{\prime}} a^{\ell_{i_{1}}+\cdots+\ell_{i_{r+1}}+s},
$$

where

$$
m^{\prime}=\left(\sum_{\substack{j \in Y \\ r \leq j \leq r+i}} m_{i_{j}}\right)+\left|\left\{j \in Y^{\prime} \mid r<j \leq r+i\right\}\right| .
$$

If $i_{r+i+1} \notin Y$, then $-\ell_{i_{1}}-\cdots-\ell_{i_{r+1}}+s_{i_{r+i+1}}<s$, and applying $a^{2} b=a$ repeatedly we obtain

$$
\begin{aligned}
& v_{r+i+1} \varphi=\left(v_{r+i} \varphi\right)\left(x_{i_{r+i+1}} \varphi\right)=b^{s}(a b)^{m^{\prime}} a^{\ell_{i}+\cdots+\ell_{i_{r+i}}+s} b^{s_{r+i+1}}(a b)^{m_{i_{r+i+1}}} a^{\ell_{i_{r+i+1}}+s_{i_{r+i+1}}} \\
& =b^{s}(a b)^{m^{\prime}} a^{\left(\ell_{t_{1}}+\cdots+\ell_{i_{r+1}}+s\right)-s_{i_{r+i+1}}+\ell_{i_{r+i+1}}+s_{i_{r+i+1}}} \\
& =b^{s}(a b)^{m^{\prime}} a^{\ell_{1}+\cdots+\ell_{i_{+}+1+1}+s} \text {. }
\end{aligned}
$$


Here

$$
m^{\prime}=\left(\sum_{\substack{j \in Y \\ r \leq j \leq r+i+1}} m_{i j}\right)+\left|\left\{j \in Y^{\prime} \mid r<j \leq r+i+1\right\}\right|
$$

since $i_{r+i+1} \notin Y$, and we conclude that again $v_{r+i+1} \varphi$ is of the required form.

We next suppose that $i_{r+i+1} \in Y$. First consider the case where $i_{r+i+1} \in Y^{\prime}$, that is, $s_{i_{r+i+1}}=\ell_{i_{1}}+\cdots+\ell_{i_{r+i}}+s>0$. Then $a^{\ell_{i_{1}}+\cdots+\ell_{i_{r+i}}+s} b^{s_{r+i+1}}=a b$ follows from a repeated application of $a^{2} b=a$, and we obtain, by (2.1),

$$
v_{r+i+1} \varphi=\left(v_{r+i} \varphi\right)\left(x_{i_{r+i+1}} \varphi\right)=b^{s}(a b)^{m^{\prime}+m_{i_{r+1+1}}+1} a^{\ell_{1}+\cdots+\ell_{i_{r+1+1}}+s},
$$

where

$$
m^{\prime}+m_{i_{r+i+1}}+1=\left(\sum_{\substack{j \in Y \\ r \leq j \leq r+i+1}} m_{i_{j}}\right)+\left|\left\{j \in Y^{\prime} \mid r<j \leq r+i+1\right\}\right|,
$$

as required. If $i_{r+i+1} \in Y-Y^{\prime}$, then $s_{i_{r+i+1}}=\ell_{i_{1}}+\cdots+\ell_{i_{r+i}}+s=0$ and

$$
\begin{aligned}
v_{r+i+1} \varphi & =\left(v_{r+i} \varphi\right)\left(x_{i_{r+i+1}} \varphi\right)=b^{s}(a b)^{m^{\prime}}(a b)^{m_{i_{r+1+i}}} a^{\ell_{i_{r+1+1}}} \\
& =b^{s}(a b)^{m^{\prime}+m_{i_{r+1+1}}} a^{\ell_{i_{1}}+\cdots+\ell_{i_{r+i+1}}+s}
\end{aligned}
$$

where

$$
m^{\prime}+m_{i_{r+i+1}}=\left(\sum_{\substack{j \in Y \\ r \leq j \leq r+i+1}} m_{i_{j}}\right)+\left|\left\{j \in Y^{\prime} \mid r<j \leq r+i+1\right\}\right|,
$$

as required. This concludes our proof by induction.

\section{Polyhedral convex cones and balanced identities}

To fix the notation we let $\mathbf{A}$ be an $m$ by $n$ matrix whose entries are integers. The rows of $\mathbf{A}$ are denoted by $\mathbf{A}_{1}, \ldots, \mathbf{A}_{m}$. Let $\mathbf{x}$ be the column vector whose entries $\mathbf{x}_{1}, \ldots, \mathbf{x}_{n}$ can assume rational values. $\mathbf{A x} \leq \mathbf{0}$ stands for the system of inequalities $\mathbf{A}_{1} \mathbf{x} \leq 0, \ldots, \mathbf{A}_{m} \mathbf{x} \leq 0$. The solution set of this system, to be interpreted as a subset of the $n$-dimensional space $\mathbb{Q}^{n}$ over the rationals, is called a polyhedral convex cone, and denoted by $\mathbf{A}^{*}$. For any subset $I$ of $M=\{1, \ldots, m\}, \mathbf{A}_{I}^{*}$ is the solution set of the system $\mathbf{A}_{i} \mathbf{x}<0, i \in I, \mathbf{A}_{i} \mathbf{x}=0, i \notin I$. The $\mathbf{A}_{I}^{*}, I \subseteq M$, are called the faces of $\mathbf{A}^{*}$. Clearly the polyhedral convex cone $\mathbf{A}^{*}$ is the disjoint union of its nonempty faces. The boundary of $\mathbf{A}^{*}$ is the face $\mathbf{A}_{n}^{*}$. We shall also be particularly interested in the face $\mathbf{A}_{M}^{*}$ which consists of the solution set of the system of strict inequalities $\mathbf{A}_{i} \mathbf{x}<0, i=1, \ldots, m$. 
The dimension of the nonempty face $\mathbf{A}_{l}^{*}$ is the dimension of the vector space which forms the solution set of the system $\mathbf{A}_{i} \mathbf{x}=0, i \notin I$. In particular, the dimension $d$ of the boundary $\mathbf{A}_{\eta}^{*}$ is $d=n-\operatorname{rank} \mathbf{A}$. The convex hull of a set $C$ of vectors is the set of all the finite linear combinations of vectors of $C$ with nonnegative coefficients. The polyhedral convex cone $\mathbf{A}^{*}$ is the convex hull of a finite number of vectors [16]. It suffices to take a set $C_{0} \cup C_{1}$ of vectors, where $C_{0}$ consists of vectors whose convex hull is the boundary $A_{\emptyset}^{*}$, and $C_{1}$ contains, for every $(d+1)$-dimensional face, a vector of that face which does not belong to the boundary. Since such representatives of the $(d+1)$-dimensional faces can be calculated, questions such as whether for a given $\mathbf{A}$ the face $\mathbf{A}_{M}^{*}$ is empty can certainly be decided. We refer to the survey paper [10] for the necessary background on polyhedral convex cones.

We now let $v \approx w$ be a balanced semigroup identity. We assume

$$
c(v)=c(w)=\left\{x_{1}, \ldots, x_{1}\right\}
$$

and write

$$
v=x_{i_{1}} \ldots x_{i_{n}} .
$$

The prefix $v_{j}$ of length $j$ of $v$ thus ends in the variable $x_{i_{j}}$. For any $1 \leq k \leq t$, we let $I_{x_{k}}=\left\{j \mid t\left(v_{j}\right)=x_{k}\right\}$, and similarly, we let $J_{x_{k}}=\left\{j \mid t\left(w_{j}\right)=x_{k}\right\}$. Hence

$$
\left|I_{x_{k}}\right|=\left|J_{x_{k}}\right|=n_{x_{k}}(v)=n_{x_{k}}(w) .
$$

For $1 \leq k \leq t$, let $j_{1}<j_{2}<\cdots<j_{n_{x_{k}}(v)}$ be the distinct elements of $I_{x_{k}}$. Let $\mathbf{V}_{x_{k}}$ be the $n_{x_{k}}(v) \times t$ matrix which has entry $n_{x_{r}}\left(v_{j_{1}-1}\right)$ in the $i$ th row and $r$ th column. In a similar fashion, we construct the matrix $\mathbf{W}_{x_{k}}$. We constructed the matrices $\mathbf{V}_{x_{k}}$ and $\mathbf{W}_{x_{k}}$ in such a way that in each column the numbers are nondecreasing. The words $v$ and $w$ can be reconstructed if the matrices $\mathbf{V}_{x_{k}}$ and $\mathbf{W}_{x_{k}}, 1 \leq k \leq t$, are given. The first row of $\mathbf{V}_{x_{k}}$ consists of only zeros if and only if $k=i_{1}$, and a similar remark applies to the matrix $\mathbf{W}_{x_{k}}$.

For $j \in I_{x_{k}}, 1 \leq k \leq t$, let $\mathbf{v}_{j}$ be the $n_{x_{k}}(v) \times t$ matrix with entry $n_{x_{r}}\left(v_{j-1}\right)$ in the $i$ th row and $r$ th column. Thus, the rows of $\mathbf{v}_{j}$ are all equal to each other, and equal to some row of $\mathbf{V}_{x_{k}}$. The matrices $\mathbf{w}_{j}$ are constructed in a similar way. We shall be interested in the polyhedral convex cones

$$
\begin{array}{ll}
\left(\mathbf{v}_{j}-\mathbf{W}_{x_{k}}\right)^{*}, & j \in I_{x_{k}}, 1 \leq k \leq t, \quad \text { and } \\
\left(\mathbf{w}_{j^{\prime}}-\mathbf{V}_{x_{k}}\right)^{*}, & j^{\prime} \in J_{x_{k}}, \quad 1 \leq k \leq t .
\end{array}
$$

In particular, we are interested in the faces of these cones which are solution sets of the systems

$$
\begin{array}{lll}
\left(L_{j}\right): & \left(\mathbf{v}_{j}-\mathbf{W}_{x_{k}}\right) \mathbf{x}<\mathbf{0}, & j \in I_{x_{k}}, \\
\left(R_{j}\right): & \left(\mathbf{w}_{j^{\prime}}-\mathbf{V}_{x_{k}}\right) \mathbf{x}<\mathbf{0}, & j^{\prime} \in J_{x_{k}},
\end{array}
$$


where $\mathbf{x}$ is the column matrix with entries $\mathrm{x}_{1}, \ldots, \mathrm{x}_{t}$. We shall use the convention that these 'unknowns' are denoted with the same letters as their corresponding variables which occur in $v$ and $w$, but we shall use a different type-face.

Polyhedral convex cones can be defined over any ordered field. Since the matrices obtained from balanced identities in the way described above are all over the integers, it makes sense to consider only the ordered field $\mathbb{Q}$ of the rationals.

The notation introduced here will be used throughout the paper.

\section{The equational theory of $B C$}

In this section we indicate how to decide, for a given identity $v \approx w$, whether or not $v \approx w$ is satisfied in $B C$. Our method yields an algorithm ready for implementation. In [23] it was mentioned that semigroup identities satisfied in $B C$ were discovered with computer assistance; unfortunately little information is given about the algorithm used. In this section we also generate many examples of identities satisfied in $B C$.

An identity $v \approx w$ which is satisfied in $B C$ is balanced because $B C$ contains a copy of the infinite cyclic semigroup. If $v=x_{i_{1}} \cdots x_{i_{n}}$, then $v^{*}=x_{i_{n}} x_{i_{n-1}} \cdots x_{i_{1}}$ is the reverse of $v$. From the presentation given for $B C$, it follows that the mapping $a \rightarrow b, b \rightarrow a$ gives rise to an antiautomorphism of $B C$, whence an identity $v \approx w$ is satisfied in $B C$ if and only if $v^{*} \approx w^{*}$ is satisfied in $B C$. From this it follows that every result concerning the identities which hold in $B C$ has its left-right dual version. The same remark applies to the semigroup $E$.

Let $v \approx w$ be an identity which is satisfied in $B C$, and let $\bar{v} \approx \bar{w}$ be the identity which results from $v \approx w$ by deleting all the occurrences of some, but not all the variables which are in $c(v)=c(w)$. Then $\bar{v} \approx \bar{w}$ is also satisfied in $B C$ since $B C$ has an identity element $a b$. We say that $\bar{v} \approx \bar{w}$ is obtained from $v \approx w$ by deletion. The fact that the equational theory of $B C$ is closed for deletion will be applied without further comment.

More specific features of the identities satisfied in $B C$ will be given later in this section. However, first we have the generalities.

THEOREM 4.1. A balanced identity $v \approx w$ is satisfied in the bicyclic semigroup $B C$ if and only if, with the notation of Section 3 , for every $1 \leq j \leq n$, the faces given by (3.5) and (3.6) of the polyhedral convex cones (3.3) and (3.4), respectively, are empty.

ProOF. We let $v \approx w$ be a balanced identity with $c(v)=c(w), v$ as in (3.1) and (3.2) and

$$
w=x_{i_{1}^{\prime}} \cdots x_{i_{n}^{\prime}} .
$$


Assume that $v \approx w$ is not satisfied in $B C$. Then there exists a substitution $\varphi$ of the variables of $c(v)=c(w)$ by elements of $B C$ such that $v \varphi \neq w \varphi$ in $B C$. Let $\varphi$ be given by (2.6). Then by Lemma 2.7,v $v=b^{s} a^{\ell_{i_{1}}+\cdots+\ell_{i_{n}}+s}$, where $s$ is given by (2.7). Similarly, $w \varphi=b^{s^{\prime}} a^{\ell_{i}+\cdots+\ell_{i_{n}^{\prime}}+s^{\prime}}$, where $s^{\prime}$ is given by

$$
s^{\prime}=\max \left(s_{i_{1}^{\prime}},-\ell_{i_{1}^{\prime}}+s_{i_{2}^{\prime}}, \ldots,-\ell_{i_{1}^{\prime}}-\cdots-\ell_{i_{n-1}^{\prime}}+s_{i_{n}^{\prime}}\right) .
$$

Since $v \approx w$ is balanced, we have

$$
\ell_{i_{1}}+\cdots+\ell_{i_{n}}=\sum_{1 \leq i \leq t} n_{\lambda_{i}}(v) \ell_{i}=\sum_{1 \leq i \leq t} n_{x_{i}}(w) \ell_{i}=\ell_{i_{1}^{\prime}}+\cdots+\ell_{i_{n}^{\prime}} .
$$

Therefore, since $v \varphi \neq w \varphi$, we have $s \neq s^{\prime}$. By symmetry we may assume that $s>s^{\prime}$. Therefore there exists $1 \leq j \leq n$ such that, for all $1 \leq j^{\prime} \leq n$

$$
-\ell_{i_{1}}-\cdots-\ell_{i_{j-}}+s_{i_{j}}>-\ell_{i_{1}^{\prime}}-\cdots-\ell_{i_{j^{\prime}-i}}+s_{i_{j^{\prime}}}
$$

Let $j \in I_{x_{k}}$, that is, $t\left(v_{j}\right)=x_{k}$ for some $1 \leq k \leq t$. Then (4.3) is satisfied for all $j^{\prime} \in J_{x_{k}}$. With $j \in I_{x_{k}}$ and $j^{\prime} \in J_{x_{k}}$ we have from (2.6) that $s_{i_{j}}=s_{k}=s_{i_{j^{\prime}}}$ since then $x_{i_{j}}=x_{k}=x_{i^{\prime},}$. Therefore (4.3) yields $\ell_{i_{1}^{\prime}}+\cdots+\ell_{i_{j^{\prime}-1}}>\ell_{i_{1}}+\cdots+\ell_{i_{j-1}}$ for all $j \in J_{x_{k}}$. Since

$$
\begin{array}{ll}
\ell_{i_{1}}+\cdots+\ell_{i_{j-1}}=\sum_{1 \leq r \leq t} n_{x_{r}}\left(v_{j-1}\right) \ell_{r}, & \text { and } \\
\ell_{i_{1}^{\prime}}+\cdots+\ell_{i_{j^{\prime}-1}}=\sum_{1 \leq r \leq t} n_{x_{r}}\left(w_{j^{\prime}-1}\right) \ell_{r}, & j^{\prime} \in J_{x_{k}},
\end{array}
$$

we see that $x_{1}=\ell_{1}, \ldots, x_{t}=\ell_{t}$ is a solution of the system $\left(L_{j}\right)$ given by (3.5). Therefore the face of (3.3) given by (3.5) is nonempty.

To prove the converse, we may by symmetry assume that there exists $1 \leq j \leq n$ such that the system $\left(L_{j}\right)$ given by (3.5) has a solution in the rationals. Then $(3.5)$ has an integral solution, say $\mathrm{x}_{1}=\ell_{1}, \ldots, \mathrm{x}_{i}=\ell_{t}$. For $r \neq k$ we choose $s_{r}=0$ if $\ell_{r} \geq 0$ and $s_{r}=-\ell_{r}$ otherwise. Thus in any case, $\ell_{r}+s_{r}=0$ and $s_{r} \geq 0$ for all $r \neq k$. We next choose $s_{k}$ such that $\ell_{k}+s_{k} \geq 0$ and

$$
s_{k}>\max _{\substack{1 \leq j^{\prime} \leq n \\ j^{\prime} \notin J_{i_{k}}}}\left(\left(\ell_{i_{1}}+\cdots+\ell_{i_{,-1}}\right)-\left(\ell_{i_{1}^{\prime}}+\cdots+\ell_{i_{j^{\prime}-1}}\right)+s_{i_{j^{\prime}}}\right) .
$$

Then (4.3) holds true for all $1 \leq j^{\prime} \leq n, j^{\prime} \notin J_{x_{k}}$. Since for $j^{\prime} \in J_{x_{k}}$ we have that $i_{j}^{\prime}=k=i_{j}$ and $\left(\ell_{1}, \ldots, \ell_{t}\right)$ is a solution of (3.5), we have that (4.3) is also satisfied for all $j^{\prime} \in J_{x_{k}}$. Thus (4.3) is satisfied for all $1 \leq j^{\prime} \leq n$. If we now define $s$ by (2.7) and $s^{\prime}$ by (4.2) we have that $s>s^{\prime}$. With the substitution $\varphi$ given as in (2.6), $v \varphi=b^{s} a^{t_{1}+\cdots+\varepsilon_{i_{n}}+s} \neq b^{s^{\prime}} a^{t_{i}+\cdots+\ell_{i_{n}}+s^{\prime}}=w \varphi$. Therefore $v \approx w$ is not satisfied in $B C$. 
REMARK 4.2. The statement of Theorem 4.1 deals more with the way the cones (3.3) and (3.4) are presented, rather than with their inherent geometric properties. Thus, the condition formulated in that statement requires that for all $1 \leq k \leq t$ and $j \in I_{x_{k}}$, either the matrix $\mathbf{v}_{j}-\mathbf{W}_{x_{k}}$ has a zero row, or the cone $\left(\mathbf{v}_{j}-\mathbf{W}_{x_{k}}\right)^{*}$ does not have a $t$-dimensional face, and likewise, for all $j^{\prime} \in J_{x_{k}}$, the matrix $\mathbf{w}_{j^{\prime}}-\mathbf{V}_{x_{k}}$ either has a zero row, or the cone $\left(\mathbf{w}_{j^{\prime}}-\mathbf{V}_{x_{k}}\right)^{*}$ does not have a $t$-dimensional face.

If one wants to verify whether a given balanced identity $v \approx w$ of length $n$ is satisfied in $B C$, then the condition of Theorem 4.1 requires us to investigate $2 n$ systems of strict inequalities. We can in practice dismiss many of the cases to be investigated. A system (3.5) or (3.6) does not have a solution if its coefficient matrix has a zero row. For systems $\left(L_{j}\right)$ given by (3.5) this is the case if and only if $j \in J_{x_{k}}$ and $v_{j} \approx w_{j}$ is balanced, and likewise for the systems $\left(R_{j}\right)$ given by (3.6). If $v=u_{1} u u_{2}, w=u_{1} u^{\prime} u_{2}, \ell\left(u_{1}\right)=p, \ell(u)=\ell\left(u^{\prime}\right)=q$, and $\ell\left(u_{2}\right)=r$, then the coefficient matrices of the systems $\left(L_{j}\right)$ and $\left(R_{j}\right)$ each contain a zero row for $j=1, \ldots, p, p+q+1, \ldots, p+q+r$, and they need not be investigated further. The greater the common prefix and suffix of $v$ and $w$, the less work remains to be done.

There is yet another way which allows us to economize on the number of cases to investigate. Suppose that $v$ contains a block of consecutive equal variables, say $x_{i_{p}} \ldots x_{i_{p+q}}$, with $x_{i_{p}}=\cdots=x_{i_{p+q}}=x_{k}$. We claim that, after investigating $\left(L_{i_{p}}\right)$ and $\left(L_{i_{p+q}}\right)$, there is no need to investigate the intermediate cases $\left(L_{i_{p+1}}\right), i \leq i<q$. Indeed, assume that the system $\left(L_{i_{p+i}}\right), 1 \leq i<q$ has a solution $\left(\ell_{1}, \ldots, \ell_{t}\right)$. If $\ell_{k} \geq 0$, then $\left(\ell_{1}, \ldots, \ell_{t}\right)$ is also a solution of $\left(L_{i_{p}}\right)$, and if $\ell_{k} \leq 0$, then $\left(\ell_{1}, \ldots, \ell_{t}\right)$ is also a solution of $\left(L_{i_{p+q}}\right)$.

EXAMPLE 4.3. We would like to verify whether the identity $v \approx w$ given by

$$
x y x x y x y^{k} x \approx x y x y x x y^{k} x,
$$

with $k \geq 1$, is satisfied in $B C$. The cone $\left(\mathbf{v}_{4}-\mathbf{W}_{x}\right)^{*}$ is the convex hull of $(-1,1)$ and $(-1,2)$. Therefore the identity $v \approx w$ is not satisfied in $B C$. So by Corollary 2.5 , every simple semigroup in the variety determined by $v \approx w$ is completely simple.

EXAMPLE 4.4. We would like to know for which values of $i, j, k, \ell$ with $0 \leq i<$ $j<\ell$ and $k \geq 1$, the identity $v \approx w$ given by

$$
x y x x^{i} y x^{\ell-i} y^{k} x \approx x y x x^{j} y x^{\ell-j} y^{k} x
$$

is satisfied in $B C$. 
The system $\left(L_{4+i}\right)$, where $x_{k}=x$, can be written as follows:

$$
\begin{array}{r}
(i+1) \mathrm{x}+\mathrm{y}<0 \\
\mathrm{x}>0 \\
(\ell-i) \mathrm{x}+\mathrm{y}>0 \\
\cdots \cdots \cdots \cdots \\
(\ell-i) \mathrm{x}+k \mathrm{y}>0
\end{array}
$$

and one verifies directly that this system has a solution unless $(k+1)(i+1) \geq$ $\ell+1$. Similarly, $\left(L_{5+i}\right)$ has no solution if and only if this condition is satisfied, and both $\left(R_{3+j}\right)$ and $\left(R_{4+j}\right)$ have no solution if and only if $\ell \geq 2 j+1$. It follows from Remark 4.2 that no other systems of strict inequalities need to be investigated.

We conclude that the given $v \approx w$ is satisfied in $B C$ if and only if $(k+1)(i+1) \geq$ $\ell+1 \geq 2(j+1)$.

From Theorem 4.1 we can clearly see that the equational theory of $B C$ is closed for deletions. Indeed, let $v \approx w$ be satisfied in $B C,|c(v)| \geq 2, x \in c(v)=c(w)$, and $\bar{v} \approx \bar{w}$ be obtained from $v \approx w$ by deleting all occurrences of $x$. Then, since the systems (3.5) and (3.6) have no solutions, they certainly have no solutions that belong to the hyperplane $\mathrm{x}=0$, and so $\bar{v} \approx \bar{w}$ is satisfied in $B C$. One cannot expect that the converse is true; even if all the identities obtained from $v \approx w$ by proper deletion are satisfied in $B C$, then it might still be so that $v \approx w$ is not satisfied in $B C$ as it is quite conceivable that one of the faces given by (3.5) or (3.6) is nonempty and disjoint from the hyperplanes $\mathrm{x}=0, x \in c(v)=c(w)$. The statement of Theorem 4.1 has its left-right dual version where suffixes of $v$ and $w$ are used instead of prefixes. That this left-right dual version is equivalent to the statement of Theorem 4.1 is true, but not immediately obvious.

There exist some interesting ways to rephrase the statement of Theorem 4.1.

THEOREM 4.5. A balanced identity $v \approx w$ is satisfied in the bicyclic semigroup $B C$ if and only if, with the notation of Section 3 , for every $1 \leq k \leq t, j \in I_{x_{k}}, j^{\prime} \in J_{x_{k}}$,

(i) $(0, \ldots, 0)$ is a linear combination, with nonnegative coefficients not all zero, of the row's of the matrix $\mathbf{v}_{j}-\mathbf{W}_{x_{i}}$, and

(ii) $(0, \ldots, 0)$ is a linear combination, with nonnegative coefficients not all zero, of the rows of the matrix $\mathbf{w}_{j^{\prime}}-\mathbf{V}_{\Lambda_{i}}$.

ProOF. From Theorem 4.1 it follows that the balanced identity $v \approx w$ is satisfied in $B C$ if and only if the systems (3.5) and (3.6) of strict inequalities are inconsistent. The above are merely reformulations of the consistency criterion found in [7] (see also [9, Theorem 6 and Corollary 5]). 
We would also like to draw the reader's attention to an inconsistency criterion due to Motzkin [17] (see also [9, Theorem 11, and subsequent remark]):

THEOREM 4.6. A balanced identity $v \approx w$ is satisfied in the bicyclic semigroup $B C$ if and only if, with the notation of Section 3, for every $1 \leq k \leq t, j \in I_{x_{k}}, j^{\prime} \in J_{x_{k}}$, the matrices $\mathbf{v}_{j}-\mathbf{W}_{x_{k}}$ and $\mathbf{w}_{j^{\prime}}-\mathbf{V}_{x_{k}}$ each satisfy the following condition: the matrix has a zero row, or one can find $q$ linearly dependent rows in the matrix which contain a $q \times(q-1)$ submatrix $B$, such that $\left(\operatorname{det} B_{\ell}\right)\left(\operatorname{det} B_{\ell+1}\right)<0$ for all $1 \leq \ell \leq q-1$, where $B_{\ell}, 1 \leq \ell \leq q$, is the matrix obtained from $B$ by deleting its $\ell$-th row.

PROOF. The proof follows immediately from Theorem 4.1 and the results mentioned just before the statement of the theorem.

COROLLARY 4.7. It is decidable whether or not, for a given balanced identity $v \approx w$, the semigroup variety determined by $v \approx w$ contains a (0-)simple semigroup which is not completely (0-)simple.

PROOF. This follows immediately from Corollary 2.5 and either one of Theorems $4.1,4.5$ or 4.6 .

At this point the reader may ask why we have expressed the condition stated in Theorem 4.1 in the language of polyhedral convex cones. After all, the conditions stated in Theorems 4.1, 4.5, and 4.6 only concern systems of strict inequalities. It will become apparent that for varieties other than the one generated by $B C$ the consideration of the entire face structure of the polyhedral convex cones is necessary. As we shall see later, this will be the case, in particular, for the variety generated by $E$. Meanwhile we have the following result which will be used later.

THEOREM 4.8. Let $v \approx w$ be an identity which is satisfied in $B C$. Then, with the notation of Section 3 ,

(i) for every $1 \leq k \leq t$ and $j \in I_{x_{k}}$, the polyhedral convex cones $\left(\mathbf{v}_{j}-\mathbf{W}_{x_{k}}\right)^{*}$ and $\left(\mathbf{v}_{j}-\mathbf{V}_{x_{k}}\right)^{*}$ coincide, and

(ii) for every $1 \leq k \leq t$ and $j^{\prime} \in J_{x_{k}}$, the polyhedral convex cones $\left(\mathbf{w}_{j^{\prime}}-\mathbf{V}_{x_{k}}\right)^{*}$ and $\left(\mathbf{w}_{j^{\prime}}-\mathbf{W}_{x_{k}}\right)^{*}$ coincide.

ProOF. Let $\left(\ell_{1}, \ldots, \ell_{t}\right)$ be in the cone $\left(\mathbf{v}_{j}-\mathbf{V}_{x_{k}}\right)^{*}$. Thus,

$$
\ell_{i_{1}}+\cdots+\ell_{i_{j-1}} \leq \ell_{i_{1}}+\cdots+\ell_{i_{r-1}}
$$

for all $r \in I_{x_{k}}$. For $i \neq k$ we choose $s_{i}$ such that $s_{i} \geq 0$ and $\ell_{i}+s_{i} \geq 0$. We next choose $s_{k}$ such that $s_{k} \geq 0, \ell_{k}+s_{k} \geq 0$ and

$$
s_{k}=\max _{m \notin I_{x_{k}}}\left(-\ell_{i_{1}}-\cdots-\ell_{i_{m-1}}+s_{i_{m}}\right)+\ell_{i_{1}}+\cdots+\ell_{i_{j-1}} .
$$


Since $s_{i,}=s_{k}=s_{i}$ for all $r \in I_{x_{k}}$, it follows from (4.4) that

$$
-\ell_{i_{1}}-\cdots-\ell_{i_{j-1}}+s_{i_{j}} \geq \max _{r \in I_{r_{k}}}\left(-\ell_{i_{1}}-\cdots-\ell_{i_{r-1}}+s_{i_{r}}\right)
$$

and so from (4.5) and (4.6) we obtain that

$$
-\ell_{i_{1}}-\cdots-\ell_{i_{j-i}}+s_{i_{1}}=\max \left(s_{i_{1}}, \ldots,-\ell_{i_{1}}-\cdots-\ell_{i_{n-1}}+s_{i_{n}}\right) .
$$

Let $\varphi$ be the substitution by elements of $B C$, given by $x_{i} \varphi=b^{s_{i}} a^{\ell_{i}+s_{i}}, 1 \leq i \leq n$. Since $v \approx w$ is satisfied in $B C$ we have by Lemma 2.7 that $v \varphi=w \varphi=b^{s} a^{\ell+s}$, where $\ell=\sum_{1 \leq i \leq t} n_{x_{i}}(v) \ell_{i}$, and $s=-\ell_{i_{1}}-\cdots-\ell_{i_{j-1}}+s_{i_{j}}$. Again by Lemma 2.7,

$$
s=\max \left(s_{i_{1}^{\prime}}, \ldots,-\ell_{i_{1}^{\prime}}-\cdots-\ell_{i_{n-1}^{\prime}}+s_{i_{n}^{\prime \prime}}\right) .
$$

In particular, $-\ell_{i_{1}}-\cdots-\ell_{i_{j-1}}+s_{i_{j}} \geq-\ell_{i_{1}^{\prime}}-\cdots-\ell_{i_{r^{\prime}-1}}+s_{i_{r^{\prime}}}$ for every $r^{\prime} \in J_{x_{k}}$. Since $s_{i,}=s_{k}=s_{i_{r^{\prime}}}$ for every $r^{\prime} \in J_{x_{k}}$, it follows that $\ell_{i_{1}}+\cdots+\ell_{i_{j-1}} \leq \ell_{i_{1}^{\prime}}+\cdots+\ell_{i_{i^{\prime}-1}}$ for every $r^{\prime} \in J_{x_{k}}$. Therefore $\left(\ell_{1}, \ldots \ell_{t}\right)$ is in the cone $\left(\mathbf{v}_{j}-\mathbf{W}_{x_{k}}\right)^{*}$. In a similar way we can prove that for $j^{\prime} \in J_{x_{k}}$, the cone $\left(\mathbf{w}_{j^{\prime}}-\mathbf{W}_{x_{k}}\right)^{*}$ is contained in the cone $\left(\mathbf{w}_{j^{\prime}}-\mathbf{V}_{x_{k}}\right)^{*}$.

Conversely, let $\left(\ell_{1}, \ldots, \ell_{l}\right)$ be in the cone $\left(\mathbf{v}_{j}-\mathbf{W}_{x_{k}}\right)^{*}$. Thus,

$$
\ell_{i_{1}}+\cdots+\ell_{i_{j-1}} \leq \ell_{i_{1}^{\prime}}+\cdots+\ell_{i_{r^{\prime}-1}^{\prime}}
$$

for all $r^{\prime} \in J_{x_{k}}$. Since $v \approx w$ is satisfied in $B C$ we have from Theorem 4.1 that the system $\left(L_{j}\right)$ given by $(3.5)$ does not have a solution. Therefore there exists $j^{\prime} \in J_{x_{k}}$ such that

$$
\ell_{i_{1}}+\cdots+\ell_{i_{j-1}}=\ell_{i_{1}}+\cdots+\ell_{i_{j^{\prime}-1}} .
$$

From (4.7) and (4.8) we have that $\ell_{i_{1}^{\prime}}+\cdots+\ell_{i^{\prime},-1} \leq \ell_{i_{1}^{\prime}}+\cdots+\ell_{i^{\prime}{ }^{\prime}-1}$ for all $r^{\prime} \in J_{k_{k}}$. However, then $\left(\ell_{1}, \ldots, \ell_{t}\right)$ is in the cone $\left(\mathbf{w}_{j^{\prime}}-\mathbf{W}_{x_{k}}\right)^{*}$, and so by the first part of the proof, also in the cone $\left(\mathbf{w}_{j},-\mathbf{V}_{x_{k}}\right)^{*}$. Thus

$$
\ell_{i_{1}}+\cdots+\ell_{i_{\jmath}-1}=\ell_{i_{1}^{\prime}}+\cdots+\ell_{i_{j^{\prime}-1}} \leq \ell_{i_{1}}+\cdots+\ell_{i_{r-1}}
$$

for all $r \in I_{x k}$. Hence $\left(\ell_{1}, \ldots, \ell_{t}\right)$ is in the cone $\left(\mathbf{v}_{j}-\mathbf{V}_{x_{k}}\right)^{*}$. In a similar way we prove that for $j^{\prime} \in J_{x_{k}}$, the cone $\left(\mathbf{w}_{j^{\prime}}-\mathbf{V}_{x_{k}}\right)^{*}$ is contained in the cone $\left(\mathbf{w}_{j^{\prime}}-\mathbf{W}_{x_{k}}\right)^{*}$.

REMARK 4.9. It is in Theorem 4.8 that for a given word $v$ the associated matrices $\mathbf{v}_{j}-\mathbf{V}_{x_{k}}$ make their first appearance; these matrices are constructed solely in terms of $v$. Therefore Theorem 4.8 could be the starting point for determining directly, for a given $v$, the words $w$ where $v \approx w$ is satisfied in $B C$, or, more modestly, for determining directly which words $v$ are isoterms in the equational theory of $B C$. We shall not pursue such an ambitious program here. Suffice it to add the following 
remark. The matrices $\mathbf{v}_{j}-\mathbf{V}_{x_{k}}$ all have a zero row. Suppose that $v \approx w$ is satisfied in $B C$. Of course $\mathbf{v}_{j}-\mathbf{W}_{x_{k}}$ need not have a zero row. If however the cone $\left(\mathbf{v}_{j}-\mathbf{V}_{x_{k}}\right)^{*}$ has a $t$-dimensional face, then it follows from Theorems 4.1 and 4.8 and Remark 4.2 that $\mathbf{v}_{j}-\mathbf{W}_{x_{k}}$ has a zero row, that is, both $v$ and $w$ have $x_{k}$ in the $j$-th position, and moreover, $v_{j} \approx w_{j}$ is balanced.

In the following we give a sufficient condition for our identity to be satisfied in $B C$. This sufficient condition proves to be very useful for generating many examples of identities $v \approx w$ which are satisfied in $B C$. In the spirit of Theorem 4.8, this sufficient condition deals with the words $v$ and $w$ separately. To the knowledge of the author, all the concrete examples of identities which are satisfied in $B C$ and have appeared in the literature satisfy this sufficient condition.

Let $v \approx w$ be a balanced identity, $v=u_{1} u u_{2}, w=u_{1} u^{\prime} u_{2}, \ell\left(u_{1}\right)=p, \ell(u)=$ $\ell\left(u^{\prime}\right)=q$, and $\ell\left(u_{2}\right)=r$. With the notation of Section 3 , for $1 \leq k \leq t, j \in I_{x_{k}}$, $p<j \leq p+q$, we let $\left(L_{j}^{\prime}\right)$ be the system of strict inequalities

$$
\left(L_{j}^{\prime}\right): \quad \sum_{1 \leq i \leq t} n_{x_{i}}\left(v_{j-1}\right) \mathrm{x}_{i}<\sum_{1 \leq i \leq t} n_{x_{i}}\left(v_{\ell-1}\right) \mathrm{x}_{i}
$$

for $\ell \in I_{x_{k}}, 1 \leq \ell \leq p, p+q+1 \leq \ell \leq p+q+r$. Likewise, for $1 \leq k \leq t, j^{\prime} \in J_{x_{k}}$, $p<j^{\prime} \leq p+q$, we let $\left(R_{j^{\prime}}^{\prime}\right)$ be the system of strict inequalities

$$
\left(R_{j^{\prime}}^{\prime}\right): \sum_{1 \leq i \leq t} n_{x_{i}}\left(w_{j^{\prime}-1}\right) \mathrm{x}_{i}<\sum_{1 \leq i \leq t} n_{x_{i}}\left(w_{\ell^{\prime}-1}\right) \mathrm{x}_{i},
$$

for $\ell^{\prime} \in J_{x_{k}}, 1 \leq \ell^{\prime} \leq p, p+q+1 \leq \ell^{\prime} \leq p+q+r$.

THEOREM 4.10. Let $v \approx w$ be a balanced identity, $v=u_{1} u u_{2}, w=u_{1} u^{\prime} u_{2}$, $\ell\left(u_{1}\right)=p, \ell(u)=\ell\left(u^{\prime}\right)=q$, and $\ell\left(u_{2}\right)=r$. If, with the notation of Section 3, for $1 \leq k \leq t, j \in I_{x_{k}}, j^{\prime} \in J_{x_{k}}, p<j, j^{\prime} \leq p+q$, the systems $\left(L_{j}^{\prime}\right)$ and $\left(R_{j}^{\prime}\right)$ given by (4.9) and (4.10), respectively, are inconsistent, then $v \approx w$ is satisfied in $B C$.

PROOF. In view of Theorem 4.1 and Remark 4.2 , in order to verify that $v \approx w$ is satisfied in $B C$, it suffices to verify that for all $1 \leq k \leq t, j \in I_{x_{k}}, j^{\prime} \in J_{x_{k}}, p<j$, $j^{\prime} \leq p+q$, the systems $\left(L_{j}\right)$ and $\left(R_{j^{\prime}}\right)$ given by (3.5) and (3.6), respectively, are inconsistent. One readily sees that for such $j\left(j^{\prime}\right)$ the strict inequalities which feature in $\left(L_{j}^{\prime}\right)\left(\left(R_{j^{\prime}}^{\prime}\right)\right)$, also feature in $\left(L_{j}\right)\left(\left(R_{j^{\prime}}\right)\right)$. Therefore if $\left(L_{j}^{\prime}\right)$ is inconsistent, then so is $\left(L_{j}\right)$, and if $\left(R_{j^{\prime}}^{\prime}\right)$ is inconsistent, then so is $\left(R_{j^{\prime}}\right)$.

EXAMPLE 4.11. The identity $v \approx w$ given by $x y x$ xy $y x^{4} y \approx x y x$ yx $y x^{4} y$ is satisfied in $B C$. To see this it suffices to verify that the systems $\left(L_{4}^{\prime}\right),\left(L_{5}^{\prime}\right),\left(R_{4}^{\prime}\right)$. and $\left(R_{5}^{\prime}\right)$ are inconsistent. For instance $\left(L_{5}^{\prime}\right)$ is inconsistent because it contains the 
inequalities $3 x+y<x$ and $3 x+y<7 x+3 y$. This identity is somewhat remarkable because it is a shortest nontrivial identity satisfied in $B C$ where one of the variables occurs only four times in each side.

The identity $v \approx w$ given by $x y y x \quad x y$ xyyx $\approx x y y x$ yx $x y y x$ is a shortest nontrivial identity satisfied in $B C$ [23]. This identity, which appeared in [1], was the first nontrivial identity found to be satisfied in $B C$. One readily verifies that the systems $\left(L_{5}^{\prime}\right),\left(L_{6}^{\prime}\right),\left(R_{5}^{\prime}\right)$, and $\left(R_{6}^{\prime}\right)$ are inconsistent.

EXAMPLE 4.12. Let $u_{n}=x_{1} \cdots x_{n}$, with $x_{1}, \ldots, x_{n}$ distinct variables. If $\psi$ is a permutation of $\{1, \ldots, n\}$, then by $u_{n}^{\psi}$ we mean $x_{1 \psi} \cdots x_{n \psi}$. Let $\psi_{1}$ and $\psi_{2}$ be permutations of $\{1, \ldots, n\}$, and $v \approx w$ the balanced identity which is of the form

$$
x u_{n}^{\psi_{1}} u_{n} u_{n} x u_{n} x x u_{n} u_{n} u_{n}^{\psi_{2}} x \approx x u_{n}^{\psi_{1}} u_{n} u_{n} x x u_{n} x u_{n} u_{n} u_{n}^{\psi_{2}} x .
$$

We can say that (4.11) is obtained from

$$
x y^{3} x y x x y^{3} x \approx x y^{3} x x y x y^{3} x
$$

via a 'scrambled' substitution. Using the sufficient condition of Theorem 4.10 it is just as easy to verify that the scrambled version (4.11) is satisfied in $B C$ as it is to verify that (4.12) is satisfied in $B C$. For instance, for (4.12), $\left(R_{7}^{\prime}\right)$ contains the inequalities $3 \mathrm{x}+3 \mathrm{y}<\mathrm{x}+\mathrm{y}$ and $3 \mathrm{x}+3 \mathrm{y}<4 \mathrm{x}+4 \mathrm{y}$ and so $\left(R_{7}^{\prime}\right)$ is inconsistent. Correspondingly, for (4.11), $\left(R_{3 n+3+i}^{\prime}\right), 1 \leq i \leq n$, contains the inequalities

$$
3 \mathrm{x}+3\left(\mathrm{x}_{1}+\cdots+\mathrm{x}_{n}\right)+\mathrm{x}_{1}+\cdots+\mathrm{x}_{i-1}<\mathrm{x}+\left(\mathrm{x}_{1}+\cdots+\mathrm{x}_{n}\right)+\mathrm{x}_{1}+\cdots+\mathrm{x}_{i-1}
$$

and

$$
3 \mathrm{x}+3\left(\mathrm{x}_{1}+\cdots+\mathrm{x}_{n}\right)+\mathrm{x}_{1}+\cdots+\mathrm{x}_{i-1}<4 \mathrm{x}+4\left(\mathrm{x}_{1}+\cdots+\mathrm{x}_{n}\right)+\mathrm{x}_{1}+\cdots+\mathrm{x}_{i-1},
$$

and so $\left(R_{3 n+3+i}^{\prime}\right)$ is inconsistent.

For $n$ even and $\psi_{1}$ and $\psi_{2}$ such that

$$
\begin{aligned}
& \left(x_{1} \cdots x_{n}\right)^{\psi:}=x_{n} x_{n-1} \cdots x_{1}, \\
& \left(x_{1} \cdots x_{n}\right)^{\psi=}=x_{2} x_{4} \cdots x_{n} x_{1} x_{3} \cdots x_{n-1},
\end{aligned}
$$

the identities (4.11) are precisely the cleverly constructed identities considered in [24]. It was proved there that every basis of the equational theory of $B C$ must contain the identities (4.11), with $n$ even and $\psi_{1}$ and $\psi_{2}$ as in (4.13).

This example shows that the sufficient condition of Theorem 4.10 together with the device of 'scrambled' substitutions allows us to generate many sophisticated identities which are satisfied in $B C$. That identities obtained by judiciously using scrambled substitutions may not be derivable, in the usual sense, from other identities that are 
satisfied in $B C$, follows from the above mentioned result of [24]. In the next section we shall use scrambled substitutions and the sufficient condition of Theorem 4.10 to find yet another infinite family of identities satisfied in $B C$, which are present in every basis of the equational theory of $B C$.

EXAMPLE 4.13. As can be expected, the sufficient condition of Theorem 4.10 is not a necessary condition. To see this, consider the identity $v \approx w$ given by

$$
x y x y x^{2 n+1} y^{2 n+1} x \approx x y x x^{j} y x^{2 n+1-j} y^{2 n+1} x,
$$

with $1 \leq j<n$. By Example 4.4 this identity is satisfied in $B C$. However, while the system $\left(R_{j+4}\right)$ is inconsistent, $\left(R_{j+4}^{\prime}\right)$ is not. It remains to be seen whether the equational theory of $B C$ contains a basis consisting of identities which satisfy the sufficient condition of Theorem 4.10.

In order to demonstrate the full power of the sufficient condition of Theorem 4.10, we give the following theorem. Many variants are possible, including a generalization to identities in more than two variables.

THEOREM 4.14. Let $u \approx u^{\prime}$ be any balanced identity involving the variables $x$ and $y$ only. Then for sufficiently large positive integers $k_{1}, k_{2}$ and $k_{3}$, the identity $v \approx w$ given by $x y x u x^{k_{1}} y^{k_{2}} x^{k_{3}} \approx x y x u^{\prime} x^{k_{1}} y^{k_{2}} x^{k_{3}}$ is satisfied in the bicyclic semigroup $B C$.

PROOF. We put $1+n_{x}(u)=1+n_{x}\left(u^{\prime}\right)=m$ and $1+n_{y}(u)=1+n_{y}\left(u^{\prime}\right)=n$. With total disregard for efficiency, we put

$$
\begin{aligned}
& k_{1}=(m+2) ! n-(m+1) \\
& k_{2}=(m+2) !(n+1) ! n-1, \\
& k_{3}=(m+2) !(n+1) ! m n-(m+2) ! n
\end{aligned}
$$

We shall verify that for such choices of $k_{1}, k_{2}$ and $k_{3}$ the systems $\left(L_{3+j}^{\prime}\right)$ and $\left(R_{3+j}^{\prime}\right)$, $3<j \leq m+n+1$, are inconsistent. Hence by the sufficient condition of Theorem 4.10 it will follow that the identity $v \approx w$ is satisfied in $B C$. We shall deal with the systems $\left(L_{3+j}^{\prime}\right)$ only, the systems $\left(R_{3+j}^{\prime}\right)$ can be dealt with in the same way.

We first assume that the prefix $v_{3+j}$ ends in the variable $y$. Then the system $\left(L_{3+j}^{\prime}\right)$ contains the strict inequalities

$$
\begin{aligned}
& \left(2+n_{x}\left(u_{j-1}\right)\right) \mathrm{x}+\left(1+n_{y}\left(u_{j-1}\right)\right) \mathrm{y}<\mathrm{x}, \\
& \left(2+n_{x}\left(u_{j-1}\right)\right) \mathrm{x}+\left(1+n_{y}\left(u_{j-1}\right)\right) \mathrm{y}<\left(m+k_{1}+1\right) \mathrm{x}+(n+\ell) \mathrm{y}
\end{aligned}
$$

for all $0 \leq \ell<k_{2}$. If we put $t=\left(m+1+k_{1}\right) /\left(2+n_{x}\left(u_{j-1}\right)\right)$, then $t$ is a positive integer such that $t>n$, and so there exists $0<\ell<k_{2}$ such that $\ell=\left(1+n_{y}\left(u_{j-1}\right)\right) t-n$. 
With this choice of $\ell,(4.15)$ becomes

$$
\left(2+n_{x}\left(u_{j-1}\right)\right) \mathrm{x}+\left(1+n_{y}\left(u_{j-1}\right)\right) \mathrm{y}<t\left(\left(2+n_{x}\left(u_{j-1}\right)\right) \mathrm{x}+\left(1+n_{y}\left(u_{j-1}\right)\right) \mathrm{y}\right),
$$

which is equivalent to

$$
\left(2+n_{x}\left(u_{j-1}\right)\right) \mathrm{x}+\left(1+n_{y}\left(u_{j-1}\right)\right) \mathrm{y}>0 .
$$

On the other hand, from (4.14), it follows that

$$
\left(1+n_{x}\left(u_{j-1}\right)\right) \mathrm{x}+\left(1+n_{y}\left(u_{j-1}\right)\right) \mathrm{y}<0
$$

is a consequence of the system $\left(L_{3+j}^{\prime}\right)$.

From (4.15) and (4.16) we have that

$$
\left(m+k_{1}+1\right) \mathrm{x}+(n+\ell) \mathrm{y}>0, \quad 0 \leq \ell<k_{2}
$$

is a consequence of the system $\left(L_{3+j}^{\prime}\right)$.

We now let $\ell=\left(m+k_{1}\right) n$. Then $0<\ell<k_{2}$ and

$$
n+\ell=(m+k+1) n>(m+k+1)(n-1),
$$

whence

$$
\frac{1+n_{x}\left(u_{j-1}\right)}{1+n_{y}\left(u_{j-1}\right)}(n+\ell)>m+k+1 .
$$

Therefore, from (4.17) it follows that

$$
\left(m+k_{1}+1\right) \mathrm{x}+(n+\ell) \mathrm{y}<0
$$

is a consequence of $\left(L_{3+j}^{\prime}\right)$ for this value of $\ell$. Confronting (4.18) with (4.19), we see that $\left(L_{3+j}^{\prime}\right)$ is inconsistent.

We next assume that the prefix $v_{3+j}$ of $v$ ends in $x$. Then the system $\left(L_{3+j}^{\prime}\right)$ contains the strict inequalities

$$
\begin{aligned}
& \left(2+n_{x}\left(u_{j-1}\right)\right) \mathrm{x}+\left(1+n_{y}\left(u_{j-1}\right)\right) \mathrm{y}<0, \\
& \left(2+n_{x}\left(u_{j-1}\right)\right) \mathrm{x}+\left(1+n_{y}\left(u_{j-1}\right)\right) \mathrm{y}<\left(m+k_{1}+\ell+1\right) \mathrm{x}+\left(n+k_{2}\right) \mathrm{y},
\end{aligned}
$$

for all $0 \leq \ell<k_{3}$. We let $s=\left(k_{2}+n\right) /\left(1+n_{y}\left(u_{j-1}\right)\right)$. Then $s$ is a positive integer, and we choose $\ell$ such that $\ell=s\left(2+n_{x}\left(u_{j-1}\right)\right)-\left(m+k_{1}+1\right)$. Then $0<\ell<k_{3}$, and for this choice of $\ell,(4.21)$ can be rewritten as

$$
\left(2+n_{x}\left(u_{j-1}\right)\right) \mathrm{x}+\left(1+n_{y}\left(u_{j-1}\right)\right) \mathrm{y}<s\left(\left(2+n_{x}\left(u_{j-1}\right)\right) \mathrm{x}+\left(1+n_{y}\left(u_{j-1}\right)\right) \mathrm{y}\right) .
$$

Confronting (4.20) with (4.22), we see that $\left(L_{3+j}^{\prime}\right)$ is inconsistent. 
In the remainder of this section we shall establish some general features of identities that are satisfied by $B C$.

The first part of the following lemma is well-known. We include it for the sake of completeness.

LEMMA 4.15. Let $v \approx w$ be a balanced identity involving the variables $x$ and $y$ only.

(i) If $v \approx w$ is satisfied in $B C$, then $h(v)=h(w)$.

(ii) If $v=x^{p} y^{q} x v^{\prime}, w=x^{p} y^{r} x w^{\prime}$, with $p \geq 0$ and $0<q<r$, then $v \approx w$ is not satisfied in $B C$.

PROOF. (i) If $h(v) \neq h(w)$, then by symmetry we may assume that $v=x^{p} y v^{\prime}$ and $w=y^{q} x w^{\prime}$ for some $p, q>0$ and some $v^{\prime}$ and $w^{\prime}$. Then the system $\left(L_{p+1}\right)$ has a solution $\mathrm{x}=-1$ and $\mathrm{y}$ sufficiently large and positive. Therefore $v \approx w$ is not satisfied in $B C$ by Theorem 4.1.

(ii) Let $v \approx w$ be as in the statement above. Then the system $\left(R_{p+r}\right)$ has a solution $\mathbf{y}=-1$ and $\mathbf{x}$ sufficiently large positive. Therefore $v \approx w$ is not satisfied in $B C$ by Theorem 4.1.

The condition of Lemma 4.15 (ii) will prove to be very useful when considering also identities in more than two variables that are satisfied in $B C$ in view of the fact that the equational theory of $B C$ is closed for deletion.

Let $\Sigma$ be an equational theory consisting of balanced identities only. Following [13] we say that $\Sigma$ is left 1-hereditary if for every $v \approx w$ of $\Sigma$, the following condition is satisfied: if $z \in c(v)=c(w)$ and $v=v_{1} z v_{2}, w=w_{1} z w_{2}$, with $z \notin c\left(v_{1}\right) \cup c\left(w_{1}\right)$, and $v_{1}$ or $w_{1}$ nonempty, then $v_{1} \approx w_{1}$ is in $\Sigma$. The notion of right 1-hereditary equational theories is defined in a dual way.

It was already shown in [24, Lemma 2] that the equational theory of $B C$ is left and right 1-hereditary. We shall give a short proof for this in the following theorem; it again follows directly from Theorem 4.1. In [20] it was found that the greatest equational theory which consists of only balanced identities and which is both left and right 1-hereditary, is precisely the equational theory which consists of all the balanced identities which are satisfied in all the orthogroups over abelian groups. This result, together with the result of [24] then yielded the consequence that the variety generated by $B C$ contains all orthogroups with abelian maximal subgroups, and thus in particular, all bands.

THEOREM 4.16 ([24]). The equational theory of $B C$ is both left and right 1-hereditary. 
PROOF. Let $v \approx w$ be any identity satisfied in $B C, z \in c(v)=c(w), v=v_{1} z v_{2}$, $w=w_{1} z w_{2}$, with $z \notin c\left(v_{1}\right) \cup c\left(w_{1}\right)$. Using deletion and Lemma 4.15 (ii), we have that $v_{1} \approx w_{1}$ is balanced or both $v_{1}$ and $w_{1}$ are empty. Let $\ell=\ell\left(v_{1}\right)=\ell\left(w_{1}\right)>0$.

With the notation of Section 3, let $1 \leq k \leq t, j \in I_{x_{k}}, j \leq \ell$. The system of inequalities

$$
\sum_{1 \leq i \leq t} n_{x_{i}}\left(v_{j-1}\right) \mathrm{x}_{i}<\sum_{1 \leq i \leq t} n_{x_{i}}\left(w_{j^{\prime}-1}\right) \mathrm{x}_{i}, \quad j^{\prime} \in J_{x_{i}}, j^{\prime} \leq \ell .
$$

is contained in the system $\left(L_{j}\right)$. Moreover, $n_{z}\left(v_{j-1}\right)=n_{z}\left(w_{j^{\prime}-1}\right)=0$ for all $j^{\prime} \in$ $J_{x_{k}}, j^{\prime} \leq \ell$. Therefore, if $(4.23)$ has a solution, then so has $\left(L_{j}\right)$ for $z$ sufficiently large positive. This however is not the case, by Theorem 4.1 and since $v \approx w$ is satisfied in $B C$. Thus the system (4.23) does not have a solution. By symmetry we may now conclude from Theorem 4.1 that $v_{1} \approx w_{1}$ is satisfied in $B C$.

We are now in the position to compare the variety $\mathbf{H S P}(B C)$ with the semigroup varieties minimal for not satisfying $[\mathcal{J}=\mathcal{D}]$.

LEMMA 4.17. Let $\mathbf{V}$ be the variety determined by all the balanced identities $v \approx w$ where $n_{x}(v)=n_{x}(w)=0$ or $n_{x}(v)=n_{x}(w) \geq 2$ for all $x \in X$, and where $h(v)=h(w), t(v)=t(w)$. Then $\mathbf{V}$ contains all the varieties minimal for not satisfying $[\mathcal{J}=\mathcal{D}]$.

ProOF. This follows immediately from [ 19 , Theorems 5.1, 5.4, 5.13, and 5.34].

COROLLARY 4.18. The variety HSP $(B C)$ properly contains the join of the varieties minimal for not satisfying $[\mathcal{J}=\mathcal{D}]$.

PROOF. It suffices to prove that $\mathbf{H S P}(B C)$ properly contains the variety $\mathbf{V}$ of the statement of Lemma 4.17. Let $v \approx w$ be an identity satisfied in $B C$. We give a proof by induction on the length $\ell$ of this identity. If $\ell=1$, then the identity is trivial and thus satisfied in $\mathbf{V}$. Let $\ell \geq 2$. If there exists no variable $z$ such that $n_{z}(v)=n_{z}(w)=1$, then $v \approx w$ is satisfied in $\mathbf{V}$ by Lemma 4.15 (i). Otherwise, $v=v_{1} z v_{2}$ and $w=w_{1} z w_{2}$, where $n_{z}(v)=n_{z}(w)=1$. By Lemma 4.15, $v_{1} \approx w_{1}$ and $v_{2} \approx w_{2}$ are satisfied in $B C$, and by the induction hypothesis they are also satisfied in $\mathbf{V}$. Therefore, again, $v \approx w$ is also satisfied in $\mathbf{V}$. Clearly $\mathbf{V}$ is then properly contained in $\operatorname{HSP}(B C)$ since $x^{2} y^{2} x \approx x y^{2} x^{2}$ is satisfied in $\mathbf{V}$ but not in $B C$.

REMARK 4.19. Corollary 4.18 shows that a semigroup variety may well not satisfy $[\mathcal{J}=\mathcal{D}]$, while all its $(0$-)simple semigroups are (0-)bisimple. If a semigroup in this variety has a $\mathcal{J}$-class that is not a $\mathcal{D}$-class, then the principal factor corresponding to this $\mathcal{J}$-class cannot be simple or 0 -simple. The semigroups $J, K, S(p), p$ prime, constructed in [19, Section 5], provide examples for this situation. 
It may be of interest to verify directly that in the variety $\mathbf{V}$ of Lemma 4.17 all simple semigroups are completely simple. Indeed, let $S$ be a simple semigroup of $\mathbf{V}$, and $a \in S$. Then $a=c a^{2} d$ for some $c, d \in S^{1}$ and so, since $S$ satisfies the identities of Lemma 4.17, $a=c a^{2} d=c^{2} a^{2} d a d=c^{3} a^{2} d a d a d=c a^{2} d a c^{2} d a d=a^{2} c^{2} d a d$. From this and its dual, it follows that $a$ is a group element of $S$. Therefore $S$ is completely simple.

\section{The axiomatic rank of the variety generated by $B C$}

It was shown by Shneerson [24] that the variety generated by $B C$ is of infinite axiomatic rank. He proved this fact by showing that every basis for the equational theory of $B C$ needs to contain the identities (4.11) of Example 4.12, with $\psi_{1}$ and $\psi_{2}$ as in (4.13). We shall prove the same, but using another family of identities. The reason why we use another family of identities is that ours has the additional nice feature that for every $k \geq 3$, there is an infinity of identities in the family which involve precisely $k$ variables.

We consider the balanced identities

$$
\begin{aligned}
& x y_{1} \cdots y_{k} x y_{1} \cdots y_{k} x x^{2} y_{1} \cdots y_{k} y_{k}^{n-2} y_{k-1}^{n-2} \cdots y_{1}^{n-2} y_{1} \cdots y_{k} x \\
& \quad \approx x y_{1} \cdots y_{k} x x y_{1} \cdots y_{k} x^{2} y_{1} \cdots y_{k} y_{k}^{n-2} y_{k-1}^{n-2} \cdots y_{1}^{n-2} y_{1} \cdots y_{k} x, \quad n \geq 3 .
\end{aligned}
$$

We can see that there are two parameters attached to such identities, namely $k$ and $n$. For $k=1$ and $n \geq 3$ this identity is satisfied in $B C$ by Example 4.4. The identities (5.1) are, for $n \geq 3$, all 'scrambled' substitutions of this simple case and the verification that they are satisfied in $B C$ is essentially as easy.

LEMMA 5.1. The identities (5.1) are satisfied in $B C$ for all $k \geq 1$ and all $n \geq 3$.

PROOF. We use the sufficient condition of Theorem 4.10. The system $\left(L_{2+k+\varepsilon}^{\prime}\right)$, $1 \leq \ell \leq k$, entails the inequalities

$$
\begin{aligned}
\mathrm{x}+\left(\mathrm{y}_{1}+\cdots+\mathrm{y}_{k}\right) & <0, \\
3 \mathrm{x}+\left(\mathrm{y}_{1}+\cdots+\mathrm{y}_{k}\right) & >0, \\
3 \mathrm{x}+n\left(\mathrm{y}_{1}+\cdots+\mathrm{y}_{k}\right) & >0,
\end{aligned}
$$

whence $\mathrm{x}>0$ and $\mathrm{x}<-\left(y_{1}+\cdots+\mathrm{y}_{n}\right)<(3 / n) \mathrm{x}$, and so $\left(L_{2+k+\ell}^{\prime}\right)$ has no solution since $n \geq 3$. $\left(L_{2 k+3}^{\prime}\right)$ has no solution for the same reason.

The systems $\left(R_{k+3}^{\prime}\right)$ and $\left(R_{3+k+\ell}^{\prime}\right), 1 \leq \ell \leq k$, each contain two inequalities which respectively entail $2 \mathrm{x}+\left(\mathrm{y}_{1}+\cdots+\mathrm{y}_{k}\right)>0$ and $2 \mathrm{x}+\left(\mathrm{y}_{1}+\cdots+\mathrm{y}_{k}\right)<0$. Therefore these systems have no solution. 
LEMMA 5.2. For $n \geq 3,1 \leq j<n-1$, the identities

$$
x \quad y_{1} y_{2} x \times y_{1} y_{2} x^{2} y_{1} y_{2} y_{2}^{n-2} y_{1}^{n-2} y_{1} y_{2} x \approx x y_{1} y_{2} x u x^{2} y_{2}^{j} y_{1} y_{2}^{n-1-j} y_{1}^{n-2} y_{1} y_{2} x,
$$

with $u=x y_{1} y_{2}$ or $u=y_{1} x y_{2}$ or $u=y_{1} y_{2} x$, are not satisfied in $B C$.

PROOF. The system $\left(R_{10+j}\right)$ has the solution $\mathrm{x}=1-n, \mathrm{y}_{1}=n-j, \mathrm{y}_{2}=-1$. Therefore the identities (5.2) are not satisfied in $B C$ by Theorem 4.10.

LEMMA 5.3. For $n \geq 3$ and words $u_{1}$ and $u_{2}$ over $\left\{y_{1}, y_{2}\right\}$ such that $n_{y_{1}}\left(u_{1}\right)=$ $n_{y_{2}}\left(u_{1}\right)=n_{y_{1}}\left(u_{2}\right)=n_{y_{2}}\left(n_{2}\right)=n$, the identities

$$
x y_{1} y_{2} x \quad y_{1} y_{2} x x^{2} u_{1} x \approx x y_{1} y_{2} x \quad y_{1} x y_{2} x^{2} u_{2} x
$$

are not satisfied in $B C$.

Proof. $\mathrm{x}=-1, \mathrm{y}_{1}=-4, \mathrm{y}_{2}=4$ is a solution for the system $\left(R_{6}\right)$. Therefore the identities (5.3) are not satisfied in $B C$ by Theorem 4.10 .

LEMMA 5.4. Let $k \geq 2$ and $n \geq 3$, and let $v \approx w$ be the identity (5.1). If $v \approx w^{\prime}$ is a nontrivial identity which is satisfied in $B C$, then $w=w^{\prime}$.

PROOF. We first consider the case $k=2$. Let $\bar{v} \approx \overline{w^{\prime}}$ be obtained from $v \approx w^{\prime}$ by deleting all the occurrences of the variable $x$, and $\overline{\bar{v}} \approx \overline{\overline{w^{\prime}}}$ obtained from $v \approx w^{\prime}$ by deleting all the occurrences of the variable $y_{2}$. Then $\bar{v} \approx \overline{w^{\prime}}$ and $\overline{\bar{v}} \approx \overline{\overline{w^{\prime}}}$ are satisfied in $B C$. Then $\overline{\bar{v}}=x y_{1} x y_{1} x x^{2} y_{1}^{n} x$, and it follows from Lemma 4.15 (ii) and its dual that $\overline{\overline{w^{\prime}}}$ has the prefix $x y_{1} x$ and the suffix $x y_{1}^{n} x$. From Example 4.4 we then have that $\overline{\overline{w^{\prime}}}=\overline{\bar{v}}$ or $\overline{\overline{w^{\prime}}}=x y_{1} x x y_{1} x^{2} y_{1}^{n} x$. In any case we see that $\overline{\overline{w^{\prime}}}$ has the suffix $x^{2} y_{1}^{n} x$. By symmetry we conclude that $w^{\prime}$ has a suffix of the form $x^{2} u_{2} x$, where $n_{y_{1}}\left(u_{2}\right)=n_{y_{2}}\left(u_{2}\right)=n$. Since $\bar{v}$ has the prefix $y_{1} y_{2} y_{1}$, so has $\overline{w^{\prime}}$ by Lemma 4.15 (ii). By symmetry we have that $w^{\prime}$ has the prefix $x y_{1} y_{2} x$.

By Lemma 4.15 (ii), both $\bar{v}$ and $\overline{w^{\prime}}$ have the suffix $y_{2} y_{1}^{n-1} y_{2}$, and therefore $u_{2}=$ $y_{2}^{j} y_{1} y_{2}^{n-1-j} y_{1}^{n-2} y_{1} y_{2}$ for some $0 \leq j<n-1$. By Lemmas 5.1 and 5.2, we need $j=0$. Therefore $w^{\prime}$ has the suffix $x^{2} y_{1} y_{2} y_{2}^{n-2} y_{1}^{n-2} y_{1} y_{2} x$. Whence $\bar{v}=\overline{w^{\prime}}$. Since the identity $v \approx w$ is nontrivial, we have from Lemma 5.3 that $w=w^{\prime}$.

We next consider the general case where $k \geq 2$. Let $1 \leq i<j \leq k$. The identity obtained from $v \approx w$ by deleting all but the variables $x, y_{i}$ and $y_{j}$ must be either trivial or of the form (5.1) by the first part of the proof. Since this holds true for all such $i$ and $j$, and since $v=w^{\prime}$ is itself nontrivial, we conclude that $w=w^{\prime}$.

THEOREM 5.5. Every basis of the equational theory of $B C$ contains the identities (5.1) for all $k \geq 2$ and $n \geq 3$. 
PROOF. Let $\Sigma$ be a basis for the equational theory of $B C$. Let $k \geq 2, n \geq 3$ and let $v \approx w$ be the identity given by (5.1). By Lemma 5.4, there exists an identity $p \approx q$ of $\Sigma$, a substitution $\sigma$, and words $u_{1}, u_{2}$ such that $v=u_{1}(p \sigma) u_{2}$ and $w=u_{1}(q \sigma) u_{2}$.

If $u \approx u^{\prime}$ is an identity that is satisfied in $B C$ and such that $u$ has the prefix $y_{1} \ldots y_{k} x y_{1}$, then by Lemma 4.15 (ii) and Theorem 4.16 , so has $u^{\prime}$. Therefore by Lemma 5.4 the word $v^{\prime}$ obtained from $v$ by deleting $h(v)$ is an isoterm in the equational theory of $B C$. Since $v \approx w$ is a nontrivial identity it therefore follows that $u_{1}$ is empty.

If $u \approx u^{\prime}$ is an identity that is satisfied in $B C$ and such that $u$ has the suffix $y_{1} \cdots y_{k} x^{3} y_{1} \cdots y_{k} y_{k}^{n-2} \cdots y_{1}^{n-2} y_{1} \cdots y_{k}$, then by Theorem 4.16 , and after using deletion and Lemma 4.15 (ii), we see that $u^{\prime}$ has the suffix $x^{3} y_{1} \cdots y_{k} y_{k}^{n-2} \ldots y_{1}^{n-2} y_{i} \cdots y_{k}$. Therefore by Lemma 5.4 the word $v^{\prime \prime}$ obtained from $v$ by deleting $t(v)$ is an isoterm in the equational theory of $B C$. Since $v \approx w$ is nontrivial, we have that $u_{2}$ is empty.

We found that $v=p \sigma, w=q \sigma$ and that $v^{\prime}$ and $v^{\prime \prime}$ are isoterms. Assume that there exists $z \in c(p)=c(q)$ such that $z \sigma$ contains the subword $x^{2}$. Then $n_{z}(p)=n_{z}(q)=1$ and $p=p_{1} z p_{2}, q=q_{1} z q_{2}$ for some words $p_{1}, p_{2}, q_{1}, q_{2}$. From Theorem 4.16 it follows that $p_{1}=q_{1}$ is satisfied in $B C$ if $p_{1}$ or $q_{1}$ is nonempty, and then $p_{1} \sigma=q_{1} \sigma$ since $v^{\prime \prime}$ is an isoterm. Thus in any case $\left(p_{1} z\right) \sigma=\left(q_{1} z\right) \sigma$ is a prefix common to $v$ and $w$. This however is impossible since $\left(p_{1} z\right) \sigma$ has the prefix $x y_{1} \cdots y_{k} x y_{1} \cdots y_{k} x^{2}$ and $w$ has not. Hence there exists no variable $z$ in $p$ such that $z \sigma$ contains the subword $x^{2}$. Since $v$ contains the subword $x^{3}$ it follows that $c(p)=c(q)$ contains a variable $x^{\prime}$ such that $x^{\prime} \sigma=x$, and then $p=p_{1}^{\prime} x^{\prime} p_{2}^{\prime}$ with $p_{1}^{\prime} \sigma=x y_{1} \cdots y_{k} x y_{1} \cdots y_{k} x$ and $p_{2}^{\prime} \sigma=x y_{1} \cdots y_{k} y_{k}^{n-2} \cdots y_{1}^{n-2} y_{1} \cdots y_{k} x$.

We shall make use of the following remark:

$$
\text { If } z \in c\left(p_{2}^{\prime}\right) \text {, then } z \in c\left(p_{1}^{\prime}\right) \text {. }
$$

Indeed, if $z \in c\left(p_{2}^{\prime}\right)$, but $z \notin c\left(p_{1}^{\prime}\right)$, then we can write $p=p_{1} z p_{2}, q=q_{1} z p_{2}$ with $z \notin c\left(p_{1}\right) \cup c\left(q_{1}\right)$, and by Theorem 4.16, $p_{1}=q_{1}$ is satisfied in $B C$. Then $p_{1} \sigma=q_{1} \sigma$ since $v^{\prime \prime}$ is an isoterm. However $p_{1} \sigma$ has the prefix $p_{1}^{\prime} \sigma$, whereas $w$ has not.

Since $p_{2}^{\prime} \sigma$ contains the subword $y_{k} y_{k}^{n-2} \cdots y_{1}^{n-2} y_{1}$, with $n \geq 3$, but $p_{1}^{\prime} \sigma$ contains neither of the subwords $y_{k}^{2}, y_{k} y_{k-1}, \ldots, y_{2} y_{1}, y_{1}^{2}$, it follows from (R) that there exist variables $y_{1}^{\prime}, \ldots, y_{k}^{\prime} \in c\left(p_{2}^{\prime}\right)$ such that $y_{1}^{\prime} \sigma=y_{1}, \ldots, y_{k}^{\prime} \sigma=y_{k}$ and moreover each of these variables occurs once or twice in $p_{1}^{\prime}$.

Assume that for some $1 \leq i \leq k$ there exists $y_{i}^{\prime \prime} \in c(p)$ such that $y_{i}^{\prime} \neq y_{i}^{\prime \prime}$ and $y_{i}^{\prime \prime} \sigma$ contains $y_{i}$. By $(\mathrm{R}), y_{i}^{\prime}$ and $y_{i}^{\prime \prime}$ each occur once in $p_{1}^{\prime}$. Let $p_{1}^{\prime \prime}$ be the shortest prefix of $p_{1}^{\prime}$ containing both $y_{i}^{\prime}$ and $y_{i}^{\prime \prime}$, and let $q_{1}^{\prime \prime}$ be the prefix of $q$ with $\ell\left(p_{1}^{\prime \prime}\right)=\ell\left(q_{1}^{\prime \prime}\right)$. By Theorem 4.16, $p_{1}^{\prime \prime} \approx q_{1}^{\prime \prime}$ is satisfied in $B C$, and since $v^{\prime \prime}$ is an isoterm we have that $p_{1}^{\prime \prime} \sigma=q_{1}^{\prime \prime} \sigma$ is a common prefix for $v$ and $w$. This, however, is impossible since $x y_{1} \ldots y_{k} x y_{1}$ is a prefix of $p_{1}^{\prime \prime} \sigma$, and $w$ does not have such a prefix.

We found that if $z \in c(p)$ such that $z \sigma$ contains $y_{i}, 1 \leq i \leq k$, then $z=y_{i}^{\prime}$. 
Therefore $\ell(z \sigma)=1$ for every $z \in c(p)$. Let $\bar{p} \approx \bar{q}$ be an identity obtained from $p \approx q$ by deleting all occurrences of $y_{2}^{\prime}, \ldots, y_{k}^{\prime}$. Then $\bar{p} \sigma=x y_{1} x y_{1} x^{3} y_{1}^{n} x$ and $\bar{q} \sigma=x y_{1} x^{2} y_{1} x^{2} y_{1}^{n} x$, where $\bar{p} \approx \bar{q}$ is satisfied in $B C$ and $\bar{p}=x_{1} y_{1}^{\prime} x_{2} y_{1}^{\prime} x_{3} x^{\prime} x_{4} y_{1}^{\prime n} x_{5}$ for variables $x_{1}, \ldots, x_{5}$, not necessarily distinct, such that $x_{i} \sigma=x$, for $i=1, \ldots, 5$. Using Lemma 4.15 (ii) and deletions one verifies that $\bar{p}$ is an isoterm unless $x_{1}=$ $x_{2}=\cdots=x_{5}=x^{\prime}$. Since $\bar{p} \sigma \neq \bar{q} \sigma, \bar{p}$ is not an isoterm. Thus, if $z \in c(p)$ such that $z \sigma$ contains $x$, then $z=x^{\prime}$.

We conclude that $\sigma$ is a bijection of $c(p)=c(q)$ onto $c(v)=c(w)$. Therefore $\Sigma$ contains $v \approx w$.

COROLLARY 5.6. Every basis of the equational theory of $B C$ contains, for every $k \geq 3$, an infinity of identities involving precisely $k$ variables.

COROLLARY 5.7 ([24]). The variety generated by BC has infinite axiomatic rank.

REMARK 5.8. Corollary 5.6 suggests that ther are several ways to introduce conditions that are stronger than the requirement to be of infinite axiomatic rank. Such distinctions are irrelevant however in the signature of monoids. In the equational theory of a monoid every identity is a consequence of an identity involving more variables. Therefore such an equational theory always has a basis, which for every $k \geq 1$ has at most one identity involving $k$ variables.

\section{The equational theory of $E$}

For a given identity $v \approx w$ we shall decide whether or not $v \approx w$ belongs to the equational theory of $E$. That $\operatorname{HSP}(E)$ contains $\operatorname{HSP}(B C)$ properly can already be seen from the following examples.

EXAMPLE 6.1. The identity $x y x x y y x^{4} y \approx x y x y x y x^{4} y$ of Example 4.11 is satisfied in $B C$, but not in $E$. The substitution $\varphi$ by elements of $E$ given by $x \varphi=a$ and $y \varphi=b$ yields $(a b)^{2} a^{3}$ for the left-hand side and $(a b)^{3} a^{3}$ for the right-hand side.

The identity $x y y x x y x y y x \approx x y y x$ yx $x y y x$ of Example 4.11 was shown in [1] to be satisfied in $B C$. This identity is not satisfied in $E$ since the substitution $\varphi$ by elements of $E$ given by $x \varphi=a$ and $y \varphi=b$ yields $b(a b) a$ for the left-hand side and $b(a b)^{2} a$ for the right-hand side.

EXAMPLE 6.2. Identities (5.1) are, for $n \geq 3$ and $k \geq 1$, not satisfied in $E$. It suffices to verify this statement for the consequence $x y^{k} x y^{k} x^{3} y^{k n} x \approx x y^{k} x^{2} y^{k} x^{2} y^{k n} x$ of (5.1) in this case. Indeed, the substitution $\varphi$ by elements of $E$ given by $x \varphi=b^{k+1} a$ and $y \varphi=a^{2}$ yields $b^{k+1}(a b) a^{(2 n-1) k-1}$ for the left-hand side and $b^{k+1}(a b)^{2} a^{(2 n-1) k+1}$ for the right-hand side. 
Following [2, Theorem 2.10] we may expect that the variety $\operatorname{HSP}(B C)$ has infinitely many covers. The following gives countably infinite concrete instances of such covers.

THEOREM 6.3. The interval $[\operatorname{HSP}(B C), \operatorname{HSP}(E)]$ in the lattice of semigroup varieties is of the power of the continuum. The variety $\mathbf{H S P}(B C)$ has infinitely many covers.

Proof. Let $A$ be the set of all identities of the form (5.1), with $n \geq 3$ and $k \geq 2$. For any subset $B$ of $A$, let $\Sigma_{B}$ be the largest equational theory contained in the equational theory of $B C$ such that $\Sigma_{B}$ does not contain the identities of $B$, and let $\mathbf{V}_{B}$ be the semigroup variety with equational theory $\Sigma_{B}$. Then $V_{\emptyset}=\operatorname{HSP}(B C)$, and $\mathbf{V}_{A} \subseteq \mathbf{H S P}(E)$ by Example 6.2. By Theorem 5.5, the interval $\left(\mathbf{V}_{y}, \mathbf{V}_{A}\right)$ consists of the varieties $\mathbf{V}_{B}, B \subseteq A$, and forms a lattice isomorphic to the lattice of all subsets of $A$.

Before we give examples of nontrivial identities satisfied in $E$, we want to characterize the identities of the equational theory of $B C$ that are satisfied in $E$.

THEOREM 6.4. A balanced identity $v \approx w$ is satisfied in the semigroup $E$ if and only if it is satisfied in the bicyclic semigroup BC and, with the notation of Section 3 ,

(i) for every $1 \leq k \leq t$ and $j \in I_{x_{k}}$, if $\left(\mathbf{v}_{j}-\mathbf{W}_{x_{k}}\right)_{A}^{*}$ and $\left(\mathbf{v}_{j}-\mathbf{V}_{x_{k}}\right)_{B}^{*}$ coincide as nonempty faces of the polyhedral convex cones $\left(\mathbf{v}_{j}-\mathbf{W}_{x_{k}}\right)^{*}$ and $\left(\mathbf{v}_{j}-\mathbf{V}_{x_{k}}\right)^{*}$, then $|A|=|B|$,

(ii) for every $1 \leq k \leq t$ and $j^{\prime} \in J_{x_{k}}$, if $\left(\mathbf{w}_{j^{\prime}}-\mathbf{V}_{x_{k}}\right)_{A}^{*}$ and $\left(\mathbf{w}_{j^{\prime}}-\mathbf{W}_{x_{k}}\right)_{B}^{*}$ coincide as nonempty faces of the polyhedral convex cones $\left(\mathbf{w}_{j^{\prime}}-\mathbf{V}_{x_{k}}\right)^{*}$ and $\left(\mathbf{w}_{j^{\prime}}-\mathbf{W}_{x_{k}}\right)^{*}$, then $|A|=|B|$.

ProOF. In the following we shall assume that $v \approx w$ is satisfied in $B C$. From Theorem 4.8 it then follows that for all $1 \leq k \leq t$ and $j \in I_{x_{k}}, j^{\prime} \in J_{x_{k}}$, the cones $\left(\mathbf{v}_{j}-\mathbf{W}_{x_{k}}\right)^{*}$ and $\left(\mathbf{v}_{j}-\mathbf{V}_{x_{k}}\right)^{*}$ coincide, and the cones $\left(\mathbf{w}_{j^{\prime}}-\mathbf{V}_{x_{k}}\right)^{*}$ and $\left(\mathbf{w}_{j^{\prime}}-\mathbf{W}_{x_{k}}\right)^{*}$ coincide.

Let us first assume that $v \approx w$ is satisfied in $E$, and let $1 \leq k \leq t$ and $j \in I_{x_{k}}$. Let $\left(\mathbf{v}_{j}-\mathbf{W}_{x_{k}}\right)_{A}^{*}$ be a nonempty face of the cone $\left(\mathbf{v}_{j}-\mathbf{W}_{x_{k}}\right)^{*}$ and $\left(\mathbf{v}_{j}-\mathbf{V}_{x_{k}}\right)_{B}^{*}$ the corresponding face in the cone $\left(\mathbf{v}_{j}-\mathbf{V}_{x_{k}}\right)^{*}$; the faces $\left(\mathbf{v}_{j}-\mathbf{W}_{x_{k}}\right)_{A}^{*}$ and $\left(\mathbf{v}_{j}-\mathbf{V}_{x_{k}}\right)_{B}^{*}$ coincide. Let $\left(\ell_{1}, \ldots, \ell_{t}\right)$ be a point in this face. For $i \neq k$ we choose $s_{i}$ such that $s_{i}>0$ and $\ell_{i}+s_{i} \geq 0$. We next choose $s_{k}$ such that

$$
\begin{aligned}
& s_{k}>0, \quad \ell_{k}+s_{k} \geq 0, \\
& s_{k}>\max _{m \notin I_{x_{k}}}\left(-\ell_{i_{1}}-\cdots-\ell_{i_{m-1}}+s_{i_{m}}\right)+\ell_{i_{1}}+\cdots+\ell_{i_{j-1}}, \\
& s_{k}>\max _{m^{\prime} \notin J_{x_{k}}}\left(-\ell_{i_{1}^{\prime}}-\cdots-\ell_{i_{m^{\prime}-1}}+s_{i_{m^{\prime}}^{\prime}}\right)+\ell_{i_{1}}+\cdots+\ell_{i_{j-1}} .
\end{aligned}
$$


We put $s=-\ell_{i_{1}}-\cdots-\ell_{i_{j-1}}+s_{i_{j}}$, where, of course, $s_{i_{j}}=s_{k}$. Since $\left(\ell_{1}, \ldots, \ell_{t}\right)$ belongs to $\left(\mathbf{v}_{j}-\mathbf{V}_{x_{k}}\right)_{B}^{*}$, we have

$$
\ell_{i_{1}}+\cdots+\ell_{i_{j-1}} \leq \ell_{i_{1}}+\cdots+\ell_{i_{r-1}}, \quad r \in I_{x_{k}}
$$

and thus, since $s_{i,}=s_{k}=s_{i,}$ for all $r \in I_{x_{k}}$,

$$
-\ell_{i_{i}}-\cdots-\ell_{i_{j-1}}+s_{i_{j}} \geq-\ell_{i_{1}}-\cdots-\ell_{i_{r-1}}+s_{i_{r}}, \quad r \in I_{x_{k}},
$$

hence $s=\max \left(s_{i_{1}}, \ldots,-\ell_{i_{1}}-\cdots-\ell_{i_{n-1}}+s_{i_{n}}\right)$.

We consider the substitution $\varphi$ by elements of $E$ given by $x_{i} \varphi=b^{s_{i}} a^{\ell_{i}+s_{i}}$ and we find that $v \varphi=w \varphi$ since $v \approx w$ is satisfied in $E$. Let $Y=\left\{j \mid s=-\ell_{i_{1}}-\cdots-\ell_{i_{j}}+s_{i_{j}}\right\}$. Then by (6.1), $Y \subseteq I_{x_{k}}$, and by (6.3) and (6.4), $|Y|=\left|I_{x_{k}}\right|-|B|$, that is, the number of equalities we find in the system (6.3). According to Lemma 2.8 we have $v \varphi=b^{s}(a b)^{\left|I_{i_{k}}\right|-|B|-1} a^{\ell_{i_{1}}+\cdots+\ell_{i_{n}}+s}$.

In a similar way we calculate $w \varphi$. Since $\left(\ell_{1}, \ldots, \ell_{t}\right)$ belongs to $\left(\mathbf{v}_{j}-\mathbf{W}_{x_{k}}\right)_{A}^{*}$ we have

$$
\ell_{i_{1}}+\cdots+\ell_{i_{1},} \leq \ell_{i_{1}^{\prime}}+\cdots+\ell_{i_{j^{\prime}-1}^{\prime}}, \quad j^{\prime} \in J_{x_{k}},
$$

and so, since $s_{i,}=s_{k}=s_{i^{\prime}}$, for all $j^{\prime} \in J_{x_{k}}$,

$$
-\ell_{i_{1}}-\cdots-\ell_{i_{j-1}}+s_{i_{j}} \geq-\ell_{i_{1}^{\prime}}-\cdots-\ell_{i_{j^{\prime}-1}}+s_{i_{j^{\prime}}}, \quad j^{\prime} \in J_{x_{k}} .
$$

In the system (6.6), precisely $\left|J_{x_{k}}\right|-|A|$ equalities occur, and since $v \approx w$ is satisfied in $B C$, we have $\left|J_{x_{k}}\right|-|A|>0$ by Theorem 4.1. By (6.2), we thus have that

$$
s=\max \left(s_{i^{\prime}}, \ldots,-\ell_{i_{1}^{\prime}}-\cdots-\ell_{i_{n-1}^{\prime}}+s_{i_{n}^{\prime}}\right) .
$$

Put $\bar{Y}=\left\{j^{\prime} \mid s=-\ell_{i_{1}^{\prime}}-\cdots-\ell_{i^{\prime}-1}+s_{i_{j}^{\prime}}\right\}$. Then by (6.2), $\bar{Y} \subseteq J_{x_{k}}$, hence $|\bar{Y}|=\left|J_{x_{k}}\right|-|A|$. According to Lemma 2.8, $w \varphi=b^{s}(a b)^{\left|J_{x_{k}}\right|-|A|-1} a^{\ell_{i_{1}}+\cdots+\ell_{i_{n}}+s}$. Since $v \varphi=w \varphi$ and since $\left|I_{x_{k}}\right|=\left|J_{x_{k}}\right|=n_{x_{k}}(v)=n_{x_{k}}(w)$, we thus have $|A|=|B|$, as required. The symmetric statement can be shown in the same way.

We proceed to prove the converse. We assume that $v \approx w$ is satisfied in $B C$ and that for every $1 \leq k \leq t$ and $j \in I_{x_{k}}$, if the faces $\left(\mathbf{v}_{j}-\mathbf{W}_{x_{k}}\right)_{A}^{*}$ and $\left(\mathbf{v}_{j}-\mathbf{V}_{x_{k}}\right)_{B}^{*}$ are nonempty and coincide, then $|A|=|B|$. Let $\varphi$ be the substitution by elements of $E$ given by (2.8). Since $v \approx w$ is satisfied in $B C$, and since (2.3) is a homomorphism from $E$ onto $B C$, we have from Lemmas 2.7 and 2.8 that $v \varphi=b^{s}(a b)^{m} a^{\ell+s}$ and $w \varphi=b^{s}(a b)^{m^{\prime}} a^{\ell+s}$, where

$$
\begin{aligned}
s & =\max \left(s_{i_{1}}, \ldots,-\ell_{i_{1}}-\cdots-\ell_{i_{n-1}}+s_{i_{n}}\right) \\
& =\max \left(s_{i_{1}^{\prime}}, \ldots,-\ell_{i_{1}^{\prime}}-\cdots-\ell_{i_{n-1}^{\prime}}+s_{i_{n}^{\prime}}\right), \\
\ell & =\ell_{i_{1}}+\cdots+\ell_{i_{n}}=\ell_{i_{n}^{\prime}}+\cdots+\ell_{i_{n}^{\prime}},
\end{aligned}
$$


and $m$ and $m^{\prime}$ are calculuated according to the statement of Lemma 2.8. It remains to be shown that $m=m^{\prime}$.

Let

$$
\begin{array}{ll}
Y=\left\{j \mid s=-\ell_{i_{1}}-\cdots-\ell_{i_{j-1}}+s_{i j}\right\}, \quad r=\min Y, \\
\bar{Y}=\left\{j^{\prime} \mid s=-\ell_{i_{1}^{\prime}}-\cdots-\ell_{i_{j^{\prime}-1}}+s_{i_{j}^{\prime}}\right\}, \quad r^{\prime}=\min \bar{Y} \\
Y^{\prime}=\left\{j \in Y \mid s_{i_{j}} \neq 0, j>r\right\}, \\
\bar{Y}^{\prime}=\left\{j^{\prime} \in \bar{Y} \mid s_{i_{j^{\prime}}} \neq 0, j^{\prime}>r^{\prime}\right\} .
\end{array}
$$

For $1 \leq k \leq t$, let $Y_{k}=Y \cap I_{x_{k}}, \bar{Y}_{k}=\bar{Y} \cap J_{x_{k}}$. Then $Y$ is the disjoint union of the nonempty sets of the form $Y_{k}$ and $\bar{Y}$ the disjoint union of the sets of the form $\bar{Y}_{k}$. Let $1 \leq k \leq t$, and assume that $Y_{k} \neq \emptyset$. For $j \in Y_{k}$ we thus have that

$$
s=-\ell_{i_{1}}-\cdots-\ell_{i_{j-1}}+s_{i_{j}} \geq-\ell_{i_{1}^{\prime}}-\cdots-\ell_{i_{j^{\prime}-1}^{\prime}}+s_{i_{j^{\prime}}^{\prime}}, \quad j^{\prime} \in J_{x_{k}},
$$

thus, since $s_{i_{j}}=s_{k}=s_{i_{j^{\prime}}}$ for all $j^{\prime} \in J_{x_{k}}$,

$$
\ell_{i_{1}}+\cdots+\ell_{i_{j-1}} \leq \ell_{i_{1}^{\prime}}+\cdots+\ell_{i_{j^{\prime}-1}^{\prime}}, \quad j^{\prime} \in J_{x_{k}},
$$

and $\left(\ell_{1}, \ldots, \ell_{t}\right)$ belongs to a face $\left(\mathbf{v}_{j}-\mathbf{W}_{x_{k}}\right)_{A}^{*}$ of the cone $\left(\mathbf{v}_{j}-\mathbf{W}_{x_{k}}\right)^{*}$. This face must coincide with a nonempty face $\left(\mathbf{v}_{j}-\mathbf{V}_{x_{k}}\right)_{B}^{*}$ of the cone $\left(\mathbf{v}_{j}-\mathbf{V}_{x_{k}}\right)^{*}$ by our assumption, and then $|A|=|B|$. We have that $\left|\bar{Y}_{k}\right|$ is the number of equalities in (6.7), that is, the number of equalities in (6.8), that is, $\left|J_{x_{k}}\right|-|A|$. Similarly, using the fact that $\left(\ell_{1}, \ldots, \ell_{t}\right)$ belongs to $\left(\mathbf{v}_{j}-\mathbf{V}_{x_{k}}\right)_{B}^{*}$ we have that $\left|Y_{k}\right|=\left|I_{x_{k}}\right|-|B|$. Therefore $\left|\bar{Y}_{k}\right|=\left|Y_{k}\right|$. By symmetry, we have that $Y_{k} \neq \varnothing$ if and only if $\bar{Y}_{k} \neq \emptyset$, and then $\left|Y_{k}\right|=\left|\bar{Y}_{k}\right|$.

Assume that $s_{i_{r}}=0$, whence $s=-\ell_{i_{1}}-\cdots-\ell_{i_{r-1}}$. If $r>1$, then

$$
\left(x_{i_{1}} \cdots x_{i_{r-1}}\right) \varphi=b^{t}(a b)^{m^{\prime \prime}} a^{\ell_{i_{1}}+\cdots+\ell_{i_{r-1}}+t}
$$

for some $m^{\prime \prime}$, with $t=\max \left(s_{i_{1}}, \ldots,-\ell_{i_{1}}-\cdots-\ell_{i_{r-2}}+s_{i_{r-1}}\right)$. Obviously $\ell_{i_{1}}+\cdots+$ $\ell_{i_{r-1}}+t \geq 0$, and since $r=\min Y, t<s$. This, however, is a contradiction. Therefore $r>1$ is impossible and instead we have $r=1, s=0$, and then $r^{\prime}=1$ since $h(v)=h(w)$ by Lemma 4.15 (i). By symmetry we have that $s_{i_{r}}=0$ if and only $s_{i^{\prime}}=0$ and if this is the case then $r=r^{\prime}$. In this circumstance,

Otherwise, $s_{i_{r}} \neq 0 \neq s_{i_{r^{\prime}}}$ and

$$
\left|Y^{\prime}\right|=\sum_{\substack{1 \leq k \leq t \\ s_{k} \neq 0}}\left|\dot{Y}_{k}\right|=\sum_{\substack{1 \leq k<t \\ s_{k} \neq 0}}\left|\bar{Y}_{k}\right|=\left|\bar{Y}^{\prime}\right| .
$$

$$
\left|Y^{\prime}\right|+1=\sum_{\substack{1 \leq k \leq t \\ s_{k} \neq 0}}\left|Y_{k}\right|=\sum_{\substack{1 \leq k \leq t \\ s_{k} \neq 0}}\left|\bar{Y}_{k}\right|=\left|\bar{Y}^{\prime}\right|+1
$$


In any case, $\left|Y^{\prime}\right|=\left|\overline{Y^{\prime}}\right|$.

From Lemma 2.8, we thus find

$$
\begin{aligned}
m & =\sum_{j \in Y} m_{i j}+\left|Y^{\prime}\right|=\sum_{1 \leq k \leq t} m_{k}\left|Y_{k}\right|+\left|Y^{\prime}\right| \\
& =\sum_{1 \leq k \leq t} m_{k}\left|\bar{Y}_{k}\right|+\left|\bar{Y}^{\prime}\right|=\sum_{j^{\prime} \in \bar{Y}} m_{i^{\prime}}+\left|\bar{Y}^{\prime}\right|=m^{\prime},
\end{aligned}
$$

and we conclude that $v \approx w$ is satisfied in $E$.

EXAMPLE 6.5. In Example 6.2 we have seen that the identity $v \approx w$ given by

$$
x y^{k} x y^{k} x^{3} y^{k n} x \approx x y^{k} x^{2} y^{k} x^{2} y^{k n} x
$$

is not satisfied in $E$. Without finding a substitution we could have found the same by applying Theorem 6.4. The cones $\left(\mathbf{w}_{k+3}-\mathbf{V}_{x}\right)^{*}$ and $\left(\mathbf{w}_{k+3}-\mathbf{W}_{x}\right)^{*}$ coincide. Their boundary consists of the point $(0,0)$ only, and they furthermore have a 1 dimensional face, namely the half line $(-k t, 2 t), t>0$. This half-line is given as a face $\left(\mathbf{w}_{k+3}-\mathbf{V}_{x}\right)_{A}^{*}=\left(\mathbf{w}_{k+3}-\mathbf{W}_{x}\right)_{B}^{*}$. with $|A|=2,|B|=3$. Therefore by Theorem 6.4, the identity is not satisfied in $E$.

REMARK 6.6. Let $v \approx w$ be a balanced identity that is satisfied in $B C$ and let $v=u_{1} u u_{2}, w=u_{1} u^{\prime} u_{2}$, with $\ell\left(u_{1}\right)=p, \ell(u)=\ell\left(u^{\prime}\right)=q$, and $\ell\left(u_{2}\right)=r$. We claim that $v \approx w$ is satisfied in $E$ if and only if for every $1 \leq k \leq t, j \in I_{x_{k}}, j^{\prime} \in J_{x_{k}}$, with $p<j \leq p+q$ and $p<j^{\prime} \leq p+q$, the conditions (i) and (ii) in the statement of Theorem 6.4 are satisfied. This certainly reduces the number of cones to be examined.

To see that our claim holds true we must revisit the converse part of the proof of Theorem 6.4. The crucial passage is the one where we prove that for $1 \leq k \leq t$, we need $\left|Y_{k}\right|=\left|\bar{Y}_{k}\right|$. If $Y_{k}$ contains a $j \in I_{x_{k}}$ such that $p<j \leq p+q$, then the same argument gives $\left|Y_{k}\right|=\left|\bar{Y}_{k}\right|$. By symmetry, if $\bar{Y}_{k}$ contains a $j^{\prime} \in J_{x_{k}}$ such that $p<j^{\prime} \leq p+q$, then $\left|Y_{k}\right|=\left|\bar{Y}_{k}\right|$. It remains to consider the case where $Y_{k}$ and $\bar{Y}_{k}$ are subsets of $\{j \mid j \leq p$ or $p+q<j\}$. In this case however $Y_{k}=\bar{Y}_{k}$. So $\left|Y_{k}\right|=\left|\bar{Y}_{k}\right|$ in all possible situations.

We can repeat the observation made in Remark 4.2; the conditions of Theorem 6.4 deal not only with the inherent geometric properties of the cones under consideration, but also with the way they are given.

Since there exists an antiautomorphism of $E$, the equational theory of $E$ is self-dual. Thus, Theorem 6.4 has its left-right dual version where suffixes are used instead of prefixes. 
COROLLARY 6.7. It is decidable whether or not, for a given balanced identity $v \approx w$, the semigroup variety determined by $v \approx w$ contains $a(0-)$ simple idempotentfree semigroup.

PROOF. This follows immediately from Corollary 2.3 and Theorem 6.4.

We give a useful sufficient condition which will enable us to generate examples ad libidum.

THEOREM 6.8. Let $v \approx w$ be a balanced identity, $v=u_{1} u u_{2}, w=u_{1} u^{\prime} u_{2}$, with $\ell\left(u_{1}\right)=p, \ell(u)=\ell\left(u^{\prime}\right)=q$, and $\ell\left(u_{2}\right)=r$. If for all $1 \leq k \leq t, j \in I_{x_{k}}, j^{\prime} \in J_{x_{k}}$, with $p<j, j^{\prime} \leq p+q$, the polyhedral convex cones $\left(\mathbf{v}_{j}-\mathbf{W}_{k_{k}}\right)^{*}$ and $\left(\mathbf{w}_{j^{\prime}}-\mathbf{V}_{x_{k}}\right)^{*}$ each coincide with their boundary, then $v \approx w$ is satisfied in $E$.

PROOF. Let $v \approx w$ be an identity that satisfies the conditions of the statement of the theorem. Then, from Theorem 4.1 and Remark 4.2, it follows that $v \approx w$ is satisfied in $B C$. Then by Theorem 4.8, for $1 \leq k \leq t$ and $j \in I_{x_{k}}, p<j \leq p+q$, the cones $\left(\mathbf{v}_{j}-\mathbf{W}_{x_{k}}\right)^{*}$ and $\left(\mathbf{v}_{j}-\mathbf{V}_{x_{k}}\right)^{*}$ coincide, and so they each coincide with their boundary. So trivially, if $\left(\mathbf{v}_{j}-\mathbf{W}_{x_{k}}\right)_{A}^{*}$ and $\left(\mathbf{v}_{j}-\mathbf{V}_{x_{k}}\right)_{B}^{*}$ are nonempty faces common to these cones, then $A=\emptyset=B$. This, together with the symmetric argument means that $v \approx w$ is satisfied in $E$ by Theorem 6.4 and Remark 6.6

We use the sufficient condition of Theorem 6.8 to construct examples of identities which are satisfied in $E$.

THEOREM 6.9. Let $u, u^{\prime}$ be words involving the variables $x$ and $y$ only, such that $\ell=\ell(u)=\ell\left(u^{\prime}\right)>0$. Let $\varphi$ be the substitution given by $x \varphi=x y, y \varphi=y x$. Then the identity $v \approx w$ given by

$$
x y^{2} x(u \varphi) x^{2} y^{3} x y^{2} x \approx x y^{2} x\left(u^{\prime} \varphi\right) x^{2} y^{3} x y^{2} x
$$

is satisfied in $E$.

Proof. One verifies that for $j \in I_{x}, 5 \leq j \leq 4+2 \ell$, the cones $\left(\mathbf{v}_{j}-\mathbf{W}_{x}\right)^{*}$ each reduce to $\{(0,0)\}$, and similarly, for $j \in I_{y}, 5 \leq j \leq 4+2 \ell$, the cones $\left(\mathbf{v}_{j}-\mathbf{W}_{y}\right)^{*}$ each reduce to $\{(0,0)\}$. By symmetry and Theorem 6.8 , the identity $v \approx w$ is satisfied in $E$. When considering the cones $\left(\mathbf{v}_{j}-\mathbf{W}_{x}\right)^{*}$ or $\left(\mathbf{v}_{j}-\mathbf{W}_{y}\right)^{*}$ one needs to distinguish the cases where $j$ is odd or $j$ is even. Thus in all, we need to examine four cases. We examine one case and leave the other three to the reader. 
Let $j \in I_{x}, 5 \leq j \leq 4+2 \ell$ and $j=2 k$ even. Then the system $\left(\mathbf{v}_{j}-\mathbf{W}_{x}\right) \mathbf{x} \leq \mathbf{0}$ entails the inequalities

$$
\begin{aligned}
& (k+1) \mathrm{x}+(k+2) \mathrm{y} \leq 0, \\
& (k+1) \mathrm{x}+(k+2) \mathrm{y} \leq \mathrm{x}+2 \mathrm{y}, \\
& (k+1) \mathrm{x}+(k+2) \mathrm{y} \leq(4+\ell) \mathrm{x}+(5+\ell) \mathrm{y}, \\
& (k+1) \mathrm{x}+(k+2) \mathrm{y} \leq(5+\ell) \mathrm{x}+(7+\ell) \mathrm{y},
\end{aligned}
$$

for which only $\mathrm{x}=0, \mathrm{y}=0$ can be a solution.

For $u \varphi=x y$ and $u^{\prime} \varphi=y x$ we find an identity of length 15 of the form (6.9) which is satisfied in $E$. Many variants of Theorem 6.9 are possible, including those involving more than two variables. We note that $E$ generates a proper semigroup variety.

PROBLEM 6.10. Does there exist a bisimple idempotent free semigroup (a congruence free idempotent free semigroup) which generates a proper semigroup variety? To put this question into perspective, we note that by [12] every idempotent free semigroup, which is left simple or right simple, generates the variety of all semigroups; furthermore, to this date we do not have any example of a bisimple idempotent free semigroup which is neither left simple nor right simple (see the concluding research problem of [5]).

\section{References}

[1] S. I. Adjan, Defining relations and algorithmic problems for groups and semigroups, Proceedings of the Steklov Institute of Mathematics 85, Translated from Russian by M. Greendlinger (Amer. Math. Soc., Providence, 1967).

[2] A. Ya. Aizenštat, 'On covers in the lattice of semigroup varieties', in: Contemporary calculus and geometry (ed. I. Ya. Bakel'man), Collection of Scientific Works (Leningrad State Pedagogical Institute, 1972) pp. 3-11 (in Russian).

[3] O. Andersen, Ein Bericht über die Struktur abstrakter Halbgruppen (Staatsexamensarbeit, Hamburg, 1952).

[4] K. Byleen, 'Embedding any countable semigroup in a 2-generated bisimple monoid', Glasgow' Math. J. 25 (1984), 153-161.

[5] __. 'Embedding any countable semigroup without idempotents in a 2-generated simple semigroup without idempotents', Glasgow Math. J. 30 (1988), 121-128.

[6] _ 'Embedding any countable semigroup in a 2-generated congruence-free semigroup', Semigroup Forum 41 (1990), 145-153.

[7] W. B. Carver, 'Systems of linear inequalities', Ann. of Math. (2) 23 (1922), 212-220.

[8] A. H. Clifford and G. B. Preston, The algebraic theory of semigroups, Math. Surveys 7 (Amer. Math. Soc. Providence, 1961 (Vol. I) and 1967 (Vol. II)). 
[9] K. Fan, 'On systems of linear inequalities', in: Linear inequalities and related systems (eds. H. W. Kuhn and A. W. Tucker), Annals of Mathematics Studies 38 (Princeton University Press, Princeton, 1956) pp. 99-156.

[10] A. J. Goldman and A. W. Tucker, 'Polyhedral convex cones', in: Linear inequalities and related systems (eds. H. W. Kuhn and A. W. Tucker), Annals of Mathematics Studies 38 (Princeton University Press, Princeton, 1956) pp. 19-40.

[11] J. M. Howie, Fundamentals of semigroup theory (Clarendon Press, Oxford, 1995).

[12] P. R. Jones, 'Analogues of the bicyclic semigroup in simple semigroups without idempotents', Proc. Roy. Soc. Edinburgh Sect. A 106A (1987), 11-24.

[13] G. I. Mashevitzky, 'On the finite basis problem for left hereditary systems of semigroup identities', in: Semigroups, automata and languages (eds. J. Almeida. G. M. S. Gomes and P. V. Silva) (World Scientific, Singapore, 1996) pp. 167-181.

[14] R. N. McKenzie, G. F. McNulty and W. F. Taylor, Algebras, lattices, varieties, Vol. I (Wadsworth \& Brooks/Cole, Monterey, 1987).

[15] L. Megyesi and G. Pollák, 'On simple principal ideal semigroups', Studia Sci. Math. Hungar. 16 (1981), 437-448.

[16] H. Minkowski, Geometrie der Zahlen (Teubner, Leipzig, 1910).

[17] T. Motzkin, Beiträge zur Theorie der linearen Ungleichungen (Azriel, Jerusalem, 1936).

[18] W. D. Munn and N. R. Reilly, 'Congruences on a bisimple $\omega$-semigroup', Proc. Glasgow Math. Assoc. 7 (1966), 184-192.

[19] F. Pastijn, 'Semigroup varieties in which some Green relations coincide', submitted.

[20] F. Pastijn and X. Yan, 'Completely regular semigroups in the variety generated by the bicyclic semigroup', Algebra Universalis 30 (1993), 234-240.

[21] S. A. Rankin and C. M. Reis, 'Semigroups with quasi-zeroes', Canad. J. Math. 32 (1980), 5I I-530.

[22] L. Rédei, 'Halbgruppen und Ringe mit Linkseinheiten ohne Linkseinselemente', Acta Math. Acad. Sci. Hungar. 11 (1960), 217-222.

[23] F. G. Schleifer, 'Looking for identities on a bicyclic semigroup with computer assistance', Semigroup Forum 41 (1990), 173-179.

[24] L. M. Schneerson, 'On the axiomatic rank of varieties generated by a semigroup or monoid with one defining relation', Semigroup Forum 39 (1989), 17-38.

[25] P. G. Trotter, 'Cancellative simple semigroups and groups', Semigroup Forum 14 (1977), 189-198.

\section{Department of Mathematics, Statistics and Computer Science}

Marquette University

Milwaukee WI 53201-1881

USA

e-mail: francisp@mscs.mu.edu 